\title{
GETTING BIOLOGISTS TO DROP ACID
}

\author{
A Thesis \\ Presented to \\ the Faculty of California Polytechnic State University, \\ San Luis Obispo
}

\author{
In Partial Fulfillment \\ of the Requirements for the Degree \\ Master of Science in Computer Science
}

by

Ryan Verdon

June 2013 
(C) 2013

Ryan Verdon

ALL RIGHTS RESERVED 


\section{Committee Membership}

TITLE:

AUTHOR:

DATE SUBMITTED:

COMMITTEE CHAIR:

COMMITTEE MEMBER:

COMMITTEE MEMBER:

COMMITTEE MEMBER:
Getting Biologists to Drop ACID

Ryan Verdon

June 2013

Dr. Alex Dekhtyar, Associate Professor of Computer Science.

Dr. Hasmik Gharibyan, Professor of Computer Science.

Science.

Dr. Anya Goodman, Associate Professor of Chemistry \& Biochemistry. 


\section{Abstract \\ Getting Biologists to Drop ACID \\ Ryan Verdon}

Bioinformatics is the field of science in which biology, computer science, and information technology merge to form a single discipline. The ultimate goal of the field is to enable the discovery of new biological insights as well as to create a global perspective from which unifying principles in biology can be discerned. Bioinformatics involves the analysis of various types of data from multiple sources to create a model of a physical process found in the real world. To do the necessary modeling, data is being piled up in various different biological databases. So far this has worked relatively well. Unfortunately the amount of biological data being generated is increasing exponentially. Currently, there are several problems with how biological data is stored. The most depressing issue comes from a survey done by Bry and Kröger in 2003. It found that out of 111 biological databases 40-44 were collections of flat files and 41-42 were relational databases. Both of these types of systems have serious scaling limits. To store all of the biological data in the future, distributed systems are needed.

There exist three types of distributed systems: Consistent Available (CA), Consistent Partition-Tolerant (CP), Available Partition-Tolerant (AP). We argue that AP systems best meet the biologists' requirements for several reasons. First, that the workloads commonly run on biological databases are reads. So heavy consistency requirements are not needed. Second, the workloads are nontransactional so there is no need for the ACID constraint commonly found in relational databases. Lastly, availability is important because research should not be hampered by long database queries. 
As a proof-of-concept application to show that an AP system works well, we needed a bioinformatics problem to tackle. Dr. Anya Goodman, a professor in the department of Chemistry and Biochemistry at Cal Poly, San Luis Obispo, offers a bioinformatics course that covers several aspects of gene annotation and genomic research. Currently there does not exist a system in which users can input and save gene annotations and get immediate feedback regarding their performance. Thus, the Community Genome Annotation Training (CGAT) database was born. In our evaluations we compared a MySQL, Couchbase, and MongoDB implementation of the CGAT back end and found that MongoDB (an AP system) performed the best for the workloads expected on $C G A T$. 


\section{Acknowledgments}

Thank you to my "funions", Eriq Augustine and Aldrin Montana, for their work on CGAT. Thanks to my friends and family for supporting me. Thanks Disneyland for rejuvenating me on the last leg of my thesis. Lastly and most importantly thank you Professor Dekthyar for being an amazing Professor and adviser. 


\section{Contents}

List of Tables $\quad$ x

List of Figures $\quad$ xi

1 Introduction 1

2 Background and Related Work $\quad 7$

2.1 Databases Background . . . . . . . . . . . . . 7

2.1.1 What is a database? . . . . . . . . . . . . 7

2.1.2 ACID transaction model ............. 7

2.1.3 BASE transaction model . . . . . . . . . . . . . . 10

2.1.4 AP Databases Evaluated for the Back End in CGAT . . . 18

2.1.5 JSON . . . . . . . . . . . . . . . . . . . 19

2.2 Biological Background . . . . . . . . . . . . . . . . . . 20

3 Dropping ACID 22

3.1 Main Observations . . . . . . . . . . . . . . . . 22

3.1.1 Flat files.................... 23

3.1.2 Complex Data. . . . . . . . . . . . . . . 23

3.1.3 Querying.................... 23

3.1.4 Workloads................... 24

3.2 Example 1: Flybase . . . . . . . . . . . . . 24

3.2.1 Database back end . . . . . . . . . . . . . 25

3.2.2 Tasks. . . . . . . . . . . . . . . . . 25

3.2.3 Problems ..................... 27

3.3 Example 2: GenBank . . . . . . . . . . . . . . . . . 27 
3.3.1 Database back end . . . . . . . . . . . . . . 28

3.3.2 Tasks. ........................ 30

3.3.3 Problems .................... 30

3.4 Conclusion . . . . . . . . . . . . . . . . 32

4 Living without ACID 33

4.1 Community Genome Annotation Training (CGAT) Database . . . 34

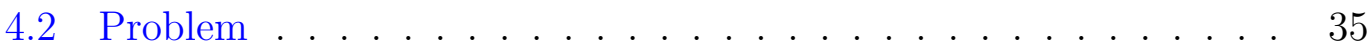

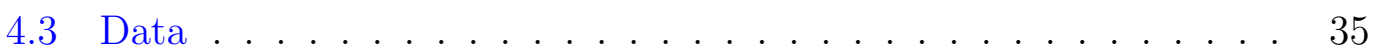

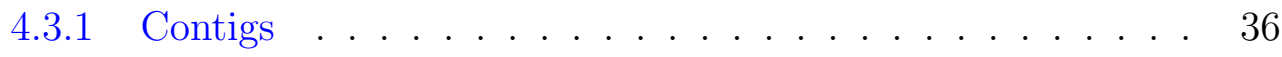

4.3.2 Annotations . . . . . . . . . . . . . . . 37

4.3 .3 Users . . . . . . . . . . . . . . . . . 37

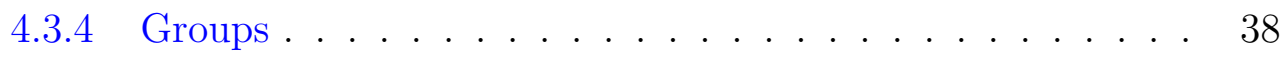

4.3.5 Help pages. . . . . . . . . . . . . . . 38

4.4 Why are CA and CP systems a bad solution for CGAT? . . . . . 39

4.5 Implementation . . . . . . . . . . . . . . . . . . . . . . . . 40

4.5.1 Website Back End . . . . . . . . . . . . . 42

4.5.2 Front End . . . . . . . . . . . . . . . . 48

4.5.3 Common Workloads . . . . . . . . . . . . . . . 48

4.5.4 Couchbase Implementation . . . . . . . . . . . . . . . . 52

5 Evaluation $\quad 53$

5.1 Experiment Design .................... 53

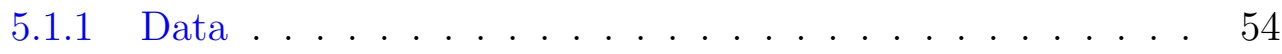

5.2 Workloads . . . . . . . . . . . . . . . 55

5.2.1 Read/Write Performance . . . . . . . . . . . . . 56

5.3 Implementation . . . . . . . . . . . . . . 56

5.4 Evaluation . . . . . . . . . . . . . . . 57

5.4 Task Assignment . . . . . . . . . . . . . . . . . 58

5.4 .2 Profile View . . . . . . . . . . . . . . . . . . 58

5.4.3 Annotation Publication . . . . . . . . . . . 58

5.4 Group Modification . . . . . . . . . . . . 59 
5.4 .5 Contig Uploads . . . . . . . . . . . . . . . . . . . . . 59

$5.4 .6 \mathrm{Read} /$ Write Performance . . . . . . . . . . . . . . . . 59

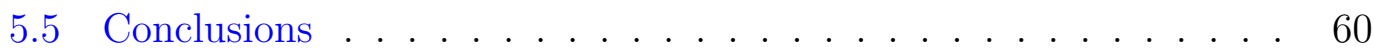

6 Conclusions and Future Work $\quad 61$

6.1 Conclusions . . . . . . . . . . . . . . . . . . . . 61

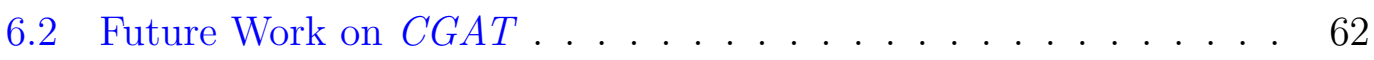

$\begin{array}{ll}\text { Bibliography } & 65\end{array}$

$\begin{array}{ll}\text { Appendices } & 69\end{array}$

A Example GenBank data file $\quad 69$

B More information on the Chado schema $\quad 71$

B.1 Chado Structure . . . . . . . . . . . . . . . . . . . . . . 71

B.2 Example graph using the Chado genetics module . . . . . . 71

$\begin{array}{lll}\text { C SQL create table statements for } C G A T & \mathbf{7 4}\end{array}$ 


\section{List of Tables}

4.1 Table of the get requests found in the CGAT API. . . . . . . . . 47

4.2 Table of the post requests found in the CGAT API. . . . . . . . . 48

5.1 Comparison of runtimes in ms for the different workloads. Each workload was run for 100000 iterations. . . . . . . . . . . . 55

5.2 Comparison of reads and writes to the same object in MySQL. . . 56

5.3 Comparison of reads and writes to the same object in Couchbase . $\quad 57$

5.4 Comparison of reads and writes to the same object in MongoDB. $\quad 57$ 


\section{List of Figures}

1.1 Growth of data inside GenBank . . . . . . . . . . . . . . . . . 3

2.1 Visual guide to the CAP theorem . . . . . . . . . . . . . 12

2.2 Example flat file . . . . . . . . . . . . . . . . . . . . . 13

2.3 Example domain name space tree . . . . . . . . . . . . 16

3.1 Flybase screenshot . . . . . . . . . . . . . . 26

3.2 GenBank screenshot . . . . . . . . . . . . . . . 31

4.1 ER Diagram of $C G A T \ldots \ldots$. . . . . . . . . . . . 41

4.2 Contig Document . . . . . . . . . . . . . . . 43

4.3 Group Document . . . . . . . . . . . . . . . . . 43

4.4 Annotation Document . . . . . . . . . . . . . . . . . . 44

4.5 User Document . . . . . . . . . . . . . . . . . . 45

4.6 Help Page Document . . . . . . . . . . . . . . . . . . 45

B.1 Chado Module Dependencies . . . . . . . . . . . . . . . . 72

B.2 Chado Genetics Module Example . . . . . . . . . . . . . . 73 


\section{Chapter 1}

\section{Introduction}

Bioinformatics is the field of science in which biology, computer science, and information technology merge to form a single discipline [1]. The ultimate goal of the field is to enable the discovery of new biological insights as well as to create a global perspective from which unifying principles in biology can be discerned [1]. Bioinformatics involves the analysis of various types of data from multiple sources to create a model of a physical process from the real world. To do the necessary modeling, data is being piled up in various biological databases. So far this has worked relatively well. Yet a problem looms on the horizon. According to Dr. Atul Butte, a Stanford professor, the amount of biological data being generated is growing exponentially [15]. He goes on to say:

"So when people think about how fast computers are getting each year, actually the data in life science is growing faster than that. In fact, we are going to reach a point where today's computers are just not even going to be able to compute on all the life science data we have [15]."

Why should anyone care that biological data will not be analyzed? Breakthroughs in research will take longer to achieve if good data is ignored or thrown 
out. Although human diseases may not be found in exactly the same form in animals, there may be sufficient data for an animal model that allows researchers to make inferences about the diseases in human beings [1]. Bioinformatics promises to rapidly create new knowledge of low-level biological processes. This, in turn, can lead to advances in the diagnosis, treatment, and prevention of many genetic diseases [1]. To be able to create new models as fast as possible, all good data gathered by researchers must be analyzed. Currently, the way the data is stored is leading to some problems in the future.

There are three major problems with how the data is stored now. The first is that the list of biological databases has exploded in number. In 2001 the list only contained 281 databases [24]. By 2008, the list had over 1000 entries [24]. That is a massive increase in the sheer number of databases storing data. Each of these databases is usually highly focused and contains data of a specific type with specific formatting [20]. Unfortunately data stored in different databases can be strongly related and dependent on each other [20]. For a biologist to get the broader context of where a piece of data fits in the grand scheme of things often requires searches of several databases [20].

The next major problem deals with how the data is stored. Bry and Kröger performed a survey in 2003 that looked at 111 biological databases to see how the data was being stored [22]. In the survey they found that of the 111 databases they sampled, 40-44 were collections of flat files and 41-42 were implemented as relational databases [22]. Both flat files and relational databases have many problems when it comes to storing biological data.

The last major problem relates to how much data is being stored in each database. This is easy to visualize by looking at a single database. For example, Figure 1.1 shows the growth of GenBank over many years. On the left axis shows 
how many base pairs were stored in GenBank for any given data on the x-axis. It is easy to see that as sequencing technology improved the amount of genomics data has exploded in volume.

\section{Growth of Sequences \& Databases}

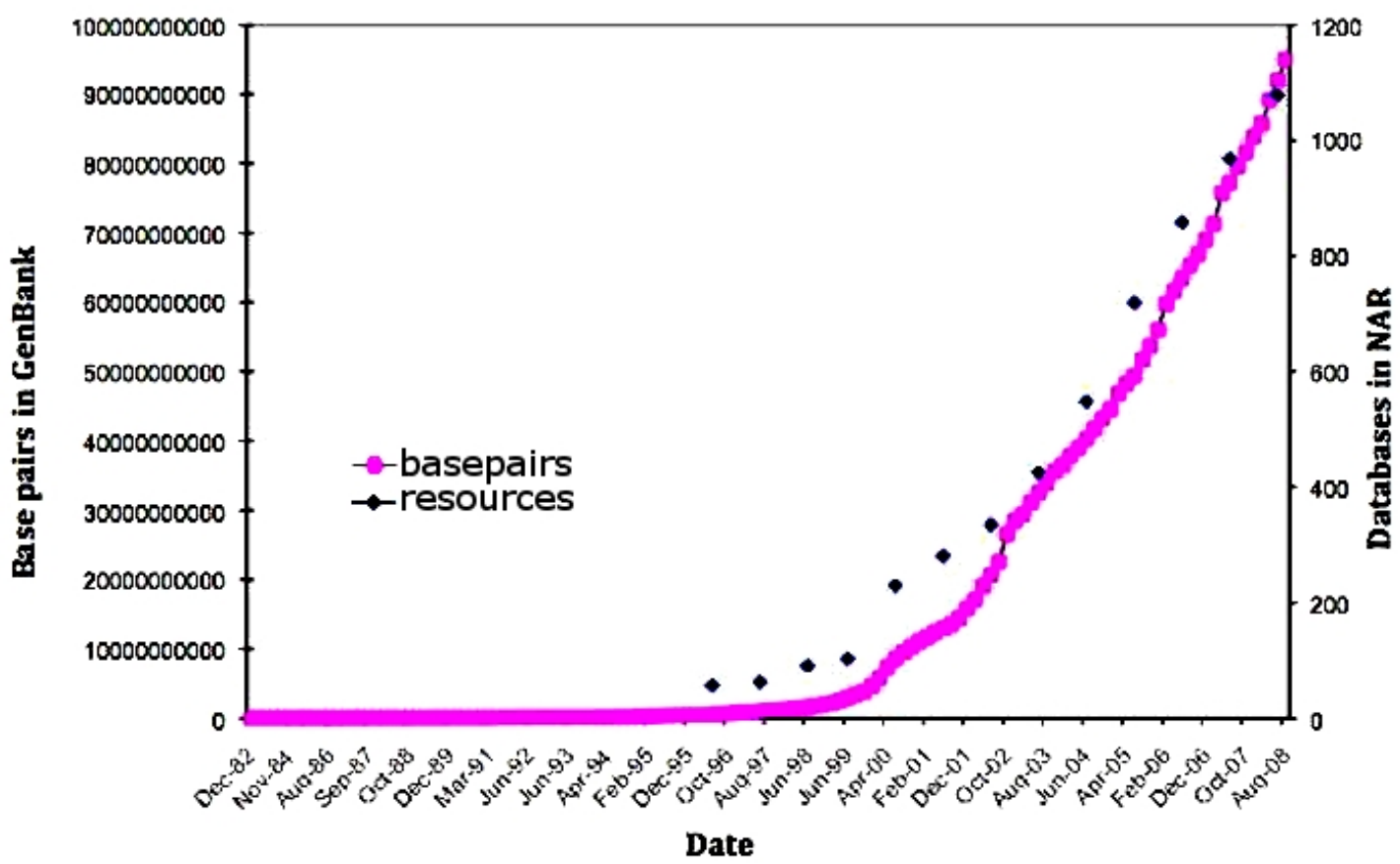

Figure 1.1: This figure depicts the amazing growth of GenBank [29].

As the volume of data increases it will be impossible to house all of the data on a single machine. To deal with all of the data, distributed systems are needed that manage data across separate computers or servers.

Recall for a moment how data is stored in computers. There exist three types of distributed systems. This fact comes from a theorem known as Brewer's Theorem, which is often referred to as the CAP Theorem [21]. The theorem presents three attributes of any distributed system, but it states that any distributed shared-data system can have at most two of them. The attributes are 
consistency, availability, and tolerance to network partitions [21]. Consistency is defined as all nodes in the distributed system seeing the same data at the same time [25]. Availability is defined as guaranteeing that every request sees a response about the successfulness of the request [25]. Tolerance to network partitions is referring to the ability of a distributed system to lose arbitrarily many messages between two nodes [25]. So the theorem leads to the three types of systems: Consistent and Available (CA), Consistent and partition tolerant $(\mathrm{CP})$, Available and partition tolerant (AP).

CA systems sacrifice the ability to lose arbitrarily many messages between two nodes in order to ensure requests can be immediately dealt with and the system is kept consistent. Usually, in CA systems availability is easily achieved because only one copy of each piece of data is stored. Since there is only one copy of each piece of data, it is impossible to not be storing the most up-to-date copy. The problem arises when a server in the database goes down. All the data stored on that server is lost until the server is restored. Unlike CA systems, CP systems sacrifice availability to ensure that they can survive one to many server failures. CP systems do this by keeping several copies of every piece of data. To guarantee consistency, on any update or inserts every copy of the data has to be reconciled before a response is sent back. This makes consistency expensive to guarantee. This also leads to a loss of immediate availability. AP systems provide the availability that $\mathrm{CP}$ systems can not guarantee, along with the ability to lose arbitrarily many messages between two nodes. AP systems do not provide strong consistency guarantees but allow for great availability by returning the first copy of data found on any query even if that given copy is old.

Now it is important to go back to the survey done by Bry and Kröger and reexamine their results using the knowledge gained from the CAP theorem. Recall 
that of the 111 databases they sampled, 40-44 were collections of flat files and 4142 were implemented as relational databases [22]. Flat files are a type of system where there is only one copy of each piece of data and the data is stored in rows like an excel file. Flat files can either be $\mathrm{CA}$ or $\mathrm{CP}$ systems depending on if the files are stored on one or several servers. Relational databases, like flat files, depending on the configuration can be either set up as CA or CP systems.

So what is wrong with CA and CP systems when it comes to storing biological data? The rapid growth of biological databases is increasing the need for each of the databases to scale up to meet the challenge. Both CA and CP systems have a hard time scaling because they have to provide full consistency on every single node in the cluster. This leads to these systems being slow and inefficient. The full consistency is not needed in many of the biological systems because updates are rare and most of the workload is reads.

Since the strong consistency constraints of CA and CP systems are not needed, there is room for AP systems to outperform CA and CP systems with biological data. With the knowledge that updates and inserts are rare occurrences, the chances that any node in the cluster has old data is extremely slim. The ability to respond quickly to any request with what will most likely be the most up-todate copy, is extremely beneficial. Also, by having the ability to tolerate network partitions, any service interruptions can be dealt with as long as the entire cluster does not become unavailable.

As a proof-of-concept application to show that an AP database solution is the best choice, we needed a bioinformatics problem to tackle. Dr. Anya Goodman, a professor in the department of Chemistry and Biochemistry at Cal Poly, San Luis Obispo, offers a bioinformatics course that covers several aspects of gene annotation and genomic research. Currently there does not exist a system in 
which users can input and save gene annotations and get immediate feedback regarding their performance. Available gene annotation software: RepeatMasker, Artemis, and others [33, 32], is not designed to provide immediate feedback for users regarding their performance. Thus, the Community Genome Annotation Training ( $C G A T)$ database was born. The main goals of $C G A T$ are to store and provide feedback on annotations performed by students, professors, and regular people across the world. All of the stored data can be then analyzed to see how effective a community is at creating new knowledge. Why is CGAT a good application to show the benefits of an AP system? There are several reasons for this. First, there is a variety of complex data being stored. Second, the workload is read-heavy. Last, the database will need to scale to thousands of users.

The main contributions of this thesis are an argument to support my position that biological databases should move to AP database solutions with the CGAT application as a case study. The rest of the thesis is structured as follows. Chapter 2 discusses background information and related work. It is followed by Chapter 3 which proposes my argument that AP databases are better equipped to deal with bioinformatics data. Chapter 4 examines a proof-of-concept bioinformatics database and shows that an AP database solution is an excellent solution for the requirements. Chapter 5 examines the evaluation of how good an AP solution was for the proof-of-concept database. Lastly, Chapter 6 discusses my contributions, conclusions, and the future work left to be done on $C G A T$. 


\section{Chapter 2}

\section{Background and Related Work}

\subsection{Databases Background}

This section contains all of the necessary background information relating to databases.

\subsubsection{What is a database?}

A database is an organized collection of data [18]. How the data is organized and accessed is based on the database management software (DBMS) being used. The main tasks of a DBMS is to accept a query, perform some internal processing, and then output the result if any.

\subsubsection{ACID transaction model}

The ACID transaction model requires very rigid requirements to be used by DBMSs to govern transactions. ACID stands for Atomicity, Consistency, 
Isolation, and Durability constraints. The goal of the ACID transaction model is to provide those constraints on every transaction. Next, it is important to understand what a transaction entails.

Transaction Definition. A transaction is a sequence of activities from a particular user that interact with the database [28]. Activities include querying, inserting, and modifying the database. It is important to note that in the user's environment a transaction is one unit of work, even though the database might have to perform several operations [28].

Now that we know what a transaction is, it is important to consider what can go wrong with a transaction and why the ACID transaction model is important for some transactions. Imagine a banking system where users have accounts. In this banking system a common task would be to move money from a users savings account to their checking account. To do this the database must first subtract the amount being transferred from the savings and then add the same amount to the users checking account. Using this example transaction, let us look at several deadly problems.

What would happen if the transaction successfully completed the subtraction from the savings account but could not successfully add to the checking account. Is this an ok situation to happen? NO! In order to prevent users from losing money, the DBMS implements the Atomicity constraint from the ACID transaction model. An atomic transaction is a transaction in which all of the activities in the transaction are run or none of them are [28]. This prevents the earlier situation from happening. If addition into the checking account cannot occur, the DBMS will undo the change to the savings account and no money will disappear from the users account. 
Let us look at another transaction where a user is moving more money from their savings account then they actually have. This would lead to their savings account balance to be a negative value. Any good banking system would require that a balance for a given account be zero or more. Is it ok if the transaction ran and left the balance as a negative value? No! The database required that the balance not be negative. To prevent transactions from leaving the database in an inconsistent state, the DBMS implements the Consistency constraint from the ACID transaction model. A consistent transaction is a transaction that leaves only legal results in the database [28]. In other words, once a transaction reaches its normal end it commits only results that are consistent with the database. In our case this would mean not leaving a balance that is negative.

Imagine a scenario in which two separate transactions are running concurrently. The first transaction is moving money from a users savings account to their checking account. The second transaction is applying a fee to any users whose balance across all of their accounts is below a threshold. If the second transaction can see the changes being made in the middle of the first transaction it could improperly charge the user a fee. Is this an ok situation? No! In order to prevent this from happening, the DBMS implements the Isolation constraint of the ACID transaction model. An isolated transaction has its effects on the database hidden from all other concurrently running transactions [28].

In another scenario, let us imagine that a transaction has just successfully added money to a user's account. If the database crashed and the results of the transaction are lost, is this ok? No! After a transaction is completed the results must be made permanent. In order to provide this, the DBMS must implement the Durability constraint of the ACID transaction model. A durable transaction is one that is guaranteed to have its results in the database survive 
any malfunctions of the database, once its results have been committed [28].

Now that we have described the definition of the ACID constraints and have looked at why they may be important, let us examine a weaker transaction model that guarantees less strict requirements but has the added benefits of being faster.

\subsubsection{BASE transaction model}

The BASE (Basically Available, Soft-state, Eventual consistency) transaction model can be summarized as weak consistency, emphasis on availability, best effort, ok to have approximate solutions, optimistic, simpler and easier to adapt [21]. The central basis for the BASE model comes from Brewer's Theorem also known as the CAP Theorem. The CAP Theorem states that any distributed shared-data system can have at most two of the following choices: consistency, availability, and tolerance to network partitions [21]. Consistency is defined as all nodes in the distributed system see the same data at the same time [25]. Availability is defined as guaranteeing that every request sees a quick response about the successfulness of the request [25]. Tolerance to network partitions is referring to the ability of a distributed system to lose arbitrarily many messages between two nodes [25].

All permutations of the three choices are useful in certain situations. For example, there exist several distributed databases and distributed file systems that use consistency and availability. These systems are generally categorized by several common traits, such as two-phase commit protocols and cache validation protocols [21]. Other distributed databases use consistency and tolerance to network partitions. These databases commonly use pessimistic locking and eliminate minority partitions [21]. Choosing which of the three choices to make 
involves examining the current problem and choosing a distributed database that makes the assumptions that the situation demands.

Figure 2.1 is a quick visual representation of the CAP theorem. It is important to realize that many of the systems listed in the figure can be configured to be two or more permutations of the CAP theorem. For example, based on the MySQL configuration, it can be either CA or CP depending on if sharding is being used or if it is in a master-slave configuration. Below there are examples given for each of the different types of distributed systems. 


\section{Visual Guide to NoSQL Systems}

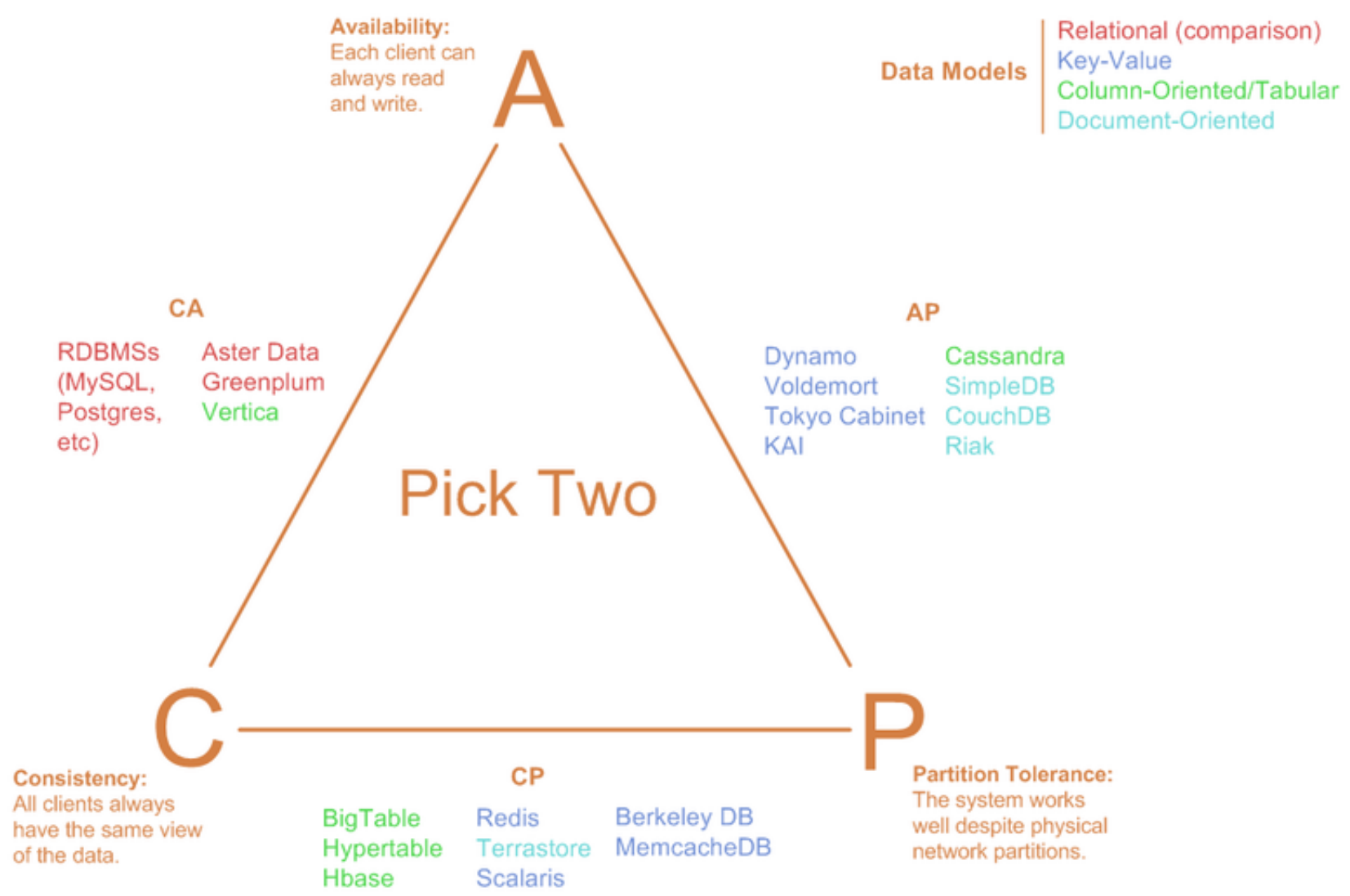

Figure 2.1: This figure depicts visually the meaning of the CAP theorem [17].

\section{Consistent Available Distributed Systems}

Consistent and available distributed systems have the ability to always respond immediately to any request from clients and the data is kept consistent. The consistency is generally achieved by only keeping one copy of the data. So there is never any old data lying around on different nodes in the cluster. The ability to tolerate network partitions is sacrificed. This is easy to see with the 
example of a two-node distributed database. If one of the nodes goes down, all of the data stored on it is gone with no way to recover it. Availability is easy to provide in CA systems because requests can be serviced as soon as they arrive at the server. There is no reconciliation process that has to keep all copies of the data consistent because there is only one copy.

Flat files. Flat files are generally plain-text or binary files that contain one record per line. The data in each record is separated by delimiters like commas or tabs. To process a flat file database, a separate program must be written and maintained to correctly perform common create, read, update, delete (CRUD) database procedures. An example flat file database of some NBA members is below.

\begin{tabular}{|lll|}
\hline id & team & last_name \\
1 & Lakers & Howard \\
2 & Lakers & Nash \\
3 & Lakers & Bryant \\
4 & Lakers & Blake \\
5 & Rockets & Brooks \\
6 & Rockets & Jones \\
7 & Rockets & Lin \\
8 & Rockets & Harden \\
& & \\
\hline
\end{tabular}

Figure 2.2: This figure depicts an example flat file containing some NBA data.

Flat files are a CA system if there is only one copy of every piece of data kept somewhere in a cluster of servers. 


\section{Consistent Partition-Tolerant Distributed Systems}

Consistent and partition tolerant systems sacrifice availability to ensure consistency across the cluster and be able to tolerate partitions. Consistency is achieved by storing a piece of data in several places across the cluster. CP systems can tolerate partitions because if a node in the cluster becomes unavailable the data can still be accessed because it is stored elsewhere in the cluster.

Relational databases. A relational database defines a set of tables which are represented by the relational data model. In the relational data model every table has a set of columns which define the attributes of the table. Every table also has a primary key which is a subset of all of the tables attributes. The primary key ensures that every row in the table is unique. Tables can relate to other tables by the use of a foreign key. Foreign keys point to the other table's primary key. There exist several flavors of relational databases. Some of the more common ones include Oracle SQL, MySQL, and Microsoft SQL server.

Generally, for most of the relational database flavors there are several ways to configure them to be either CA or CP. For this example, looking at MySQL, if the cluster is configured to store a complete copy of every table on each server in the cluster, than the relational database is a CP system. This example depicts a CP system because partition tolerance is achieved by storing a copy of every piece of data on every server in the cluster. No matter which node is queried, the same data is returned. If a server goes down there is no effect on the cluster because the data can still be read because it is copied on every server in the cluster. Availability is lost since every update, insert, or delete requires changes on every node in the cluster which can take a long time. 


\section{Available Partition-Tolerant Distributed Systems}

Available and partition-tolerant distributed systems sacrifice consistency for increased throughput. Multiple copies of every piece of data are stored because of the partition-tolerance constraint. To get the increased throughput, AP systems give up consistency in order to service requests faster. To guarantee availability, AP systems return the first copy of the data that is found. The given copy might be old or it might be the most up-to-date. AP systems run a process in the background known as asynchronous updates to ensure that all copies of the data eventually are consistent. 


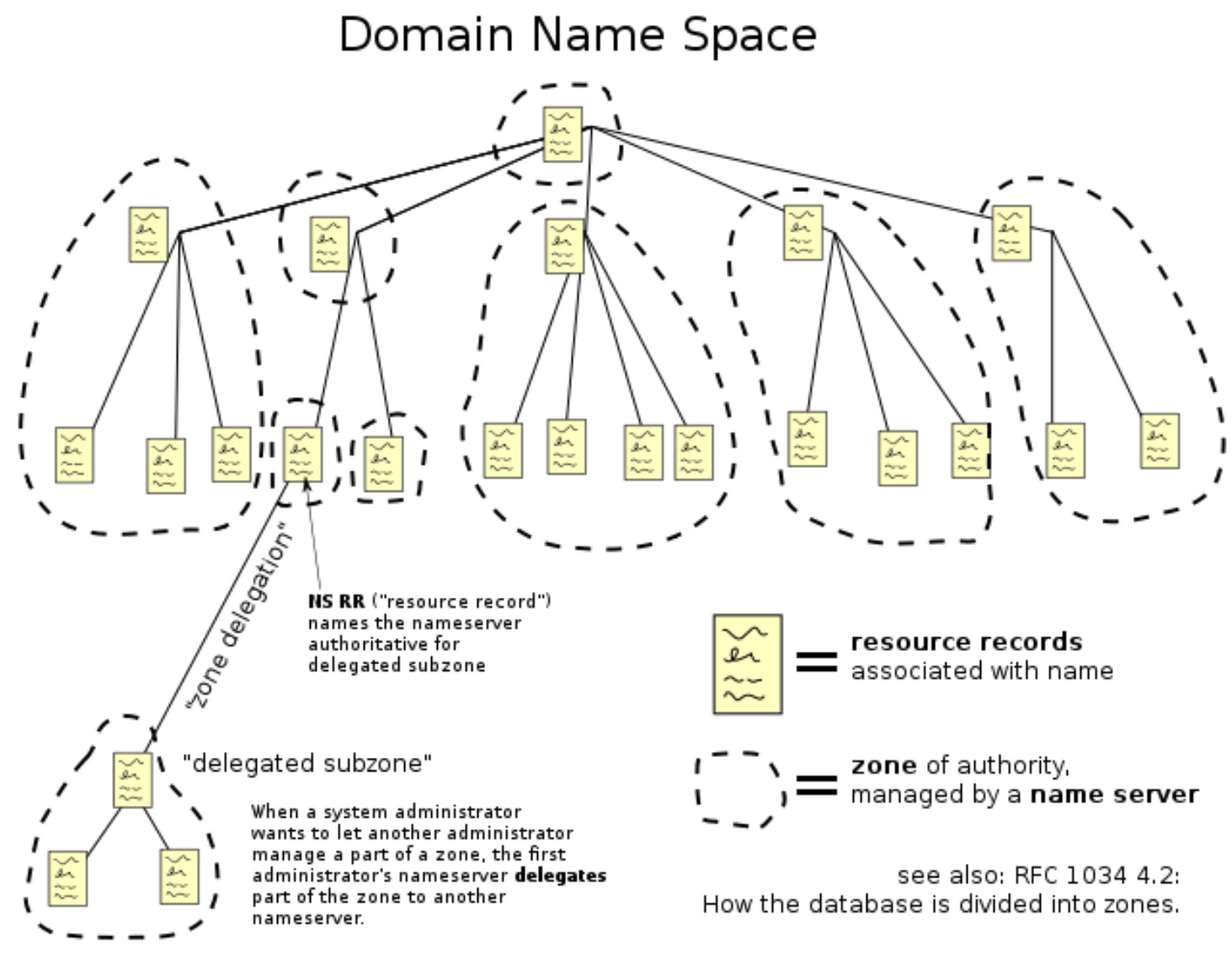

Figure 2.3: This figure depicts an example domain name space tree [4].

Domain Name System. The Domain Name System (DNS) is a hierarchical distributed naming system for computers, services, or any resource connected to the Internet or a private network [4]. DNS associates several types of information to domain names. Most commonly, DNS translates domain names into IP addresses. DNS is organized via the Domain Name Space tree. An example Domain Name Space tree is depicted in Figure 2.3. The Domain Name Space is a tree of domain names which starts at the root zone. Each node in the tree contains zero or more resource records which hold information about the domain name. The 
tree is sub-divided into DNS zones. A DNS zone contains one or more domain names.

It is easy to see that DNS is an AP distributed system by examining how domain names are converted into IPs. For this example, the fictional domain name www.ryanv.com is located at 192.168.0.1. When someone on the Cal Poly wireless network goes to www.ryanv.com, their browser goes to the Cal Poly DNS server to locate www.ryanv.com. The Cal Poly DNS server does not know about www.ryanv.com because it is not in its DNS zone. So it contacts the root DNS to locate www.ryanv.com. The root server does not know where www.ryanv.com is located but it knows the IP of the .com DNS server. So the Cal Poly DNS server now asks the .com domain name server where www.ryanv.com is. The .com server knows the IP of www.ryanv.com is 192.168.0.1 because the domain name is in its DNS zone.

With this knowledge of how DNS resolves domain names, let us look at how consistency is not provided. DNS relies heavily on caching because performing the full domain name resolution is an expensive task. So after the first time www.ryanv.com is resolved to 192.168.0.1, the Cal Poly domain server will cache the result. Let us imagine a case where a user goes to www.ryanv.com successfully so the Cal Poly DNS server will cache the IP address. There is a chance that the IP address of www.ryanv.com will change so while the old IP is cached on the Cal Poly DNS server, the data will be inconsistent with the real data.

\section{CAP theorem revisited}

The main thing to take away from the CAP theorem is that consistency is expensive. Consistency requires keeping multiple copies of every piece of data 
up-to-date on all updates, inserts, and deletes. By not having to keep all copies of the data up-to-date, higher throughput of requests can be maintained.

\subsubsection{AP Databases Evaluated for the Back End in $C G A T$}

\section{MongoDB}

MongoDB is a general purpose open-source AP database [14]. MongoDB has the following major features:

- Document data model with dynamic schemas

- Full, flexible index support and rich queries

- Auto-Sharding for horizontal scalability

- Built-in replication for high availability

MongoDB is a document store type of AP database. Using basic JSON (described in the next section) features, complex data-structure otherwise known as documents can be constructed with nested documents inside [14]. Using MongoDB's query language, complex queries can be made that can access fields nested deep inside of documents. MongoDB sacrifices consistency when distributed over several servers in order to provide quick querying capabilities.

\section{Couchbase}

Couchbase is an open-source AP database for interactive web applications and mobile applications [3]. Couchbase has the following major features:

- Flexible data model using JSON to represent documents 
- Map-reduce for large aggregate queries

- Auto-Sharding and other cross cluster cloning capabilities

- Can create primary and secondary indexes on documents for fast querying

Couchbase is a document store type of AP database. At the time the evaluations were run, we were unable to use common document store database features like being able to query on fields within a document. This is because the document store features were not fully implemented yet. Anyways, key-value stores provide a get and put interface. get(key) retrieves a value from the given key. put(key, value) associates the value with the key provided to put.

\subsubsection{JSON}

JSON (JavaScript Object Notation) is an open standard designed for humanreadable data interchange [13]. JSON is derived from JavaScript. It is used to represent simple data structures with associative arrays known as objects. JSON is often used for serializing and transmitting structured data over the Internet as an alternative to XML.

JSON's basic types include:

- Number

- String

- Boolean

- Array - an ordered sequence of values 
- Object - an unordered collection of key:value pairs with the ':' character separating the key and the value

- null

The example below shows the JSON representation of an object that describes a person. The object contains string fields for first and last names, a number for age, an object representing the person's address and an array of phone number objects.

\{

"firstName": "Bob",

"lastName": "Smith",

"age" : 21,

"phoneNumbers": [

\{

"type": "home",

"number": "(123) 456-7890"

\} ,

\{

"type": "mobile",

"number": "(123) 456-7890"

\}

],

"address": \{

"street": "123 Fake Street",

"city": "Fake City",

"state": "CA",

"zip": 12345

\}

\}

\subsection{Biological Background}

Genomics research attempts to understand information contained in the genomes of various organisms. The genome is the entire DNA sequence that encodes in- 
formation and drives all cellular processes in an organism. However, due to the size of genomes, biologists are only able to sequence sections, or fragments, of a genome at a time. One or more contiguous fragments of the genome together form a contig, or simply a contiguous sequence of DNA nucleotides.

The genome contains several components, or sub-regions, that have different functions or roles. These components describe in detail how a genome is decoded to drive cellular processes. Annotations - the process of linking biological information of the organism to the genome - are an important step to being able to relate information learned from the sequence back to broader understandings of the associated organism and, ideally, related organisms [34].

Again, since the genome is so large, biologists typically annotate sections of the genome, specifically genes. A gene is a section of the genome that codes for a functional protein used for biological processes inside and outside of the cell. A gene contains several components, or sub-regions, that have different functions or roles-exons, introns, and others. These components that are located in a gene are some of the most basic but most important pieces of information that must be annotated in order to be able to make meaningful biological interpretations from information learned from the genomic sequence. 


\section{Chapter 3}

\section{Dropping ACID}

I believe that most biological databases should be using AP systems for the back end. This is currently not the case. To attempt to substantiate my position, general observations are presented that come from having examined many biological databases. Then concrete examples of biological databases are shown that match my observations. The example biological databases are evaluated by examining the common tasks performed and by evaluating the fit of the database back end.

\subsection{Main Observations}

From my research I have noted four main observations. Each is discussed in turn below. 


\subsubsection{Flat files}

There is a surprising amount of flat file databases still being used by biologists. Some of the databases are extremely large in size as well. To use the data faster and more efficiently, some projects parse the flat file databases into relational databases so that faster querying can be done.

\subsubsection{Complex Data}

The data found in biological databases is extremely varied and complex. Types of data can range from long strands of DNA to images from medical scanners. Not only is the data complex but the relationships between the data are often complex as well. Frequently, any piece of data can be related to numerous other pieces of data in the same database as well as data in other completely different databases. A good example of data that is commonly not in the same database are research publications. Normally, when data is uploaded, a PubMed article ID is stored with it.

With the complex data comes complex metadata. Complex metadata includes who uploaded the data, when it was uploaded, who touched the data last, when the data was touched last, what changes have been made to the data since it was first uploaded, and more.

\subsubsection{Querying}

From my research, there was very little use of ad-hoc queries. Most of the queries were done through web forms or APIs set up by the developers. The vast majority of queries were key lookups or index-based lookups. For example, given 
a PubMed article ID, the system looks up the article. This was an extremely common practice with all types of data. Given the data's ID find the data.

\subsubsection{Workloads}

The vast majority of the workloads run on the biological databases are reads. Updates and inserts tend to happen in large chunks and happen daily or several times a year.

\subsection{Example 1: Flybase}

Flybase is the primary biology database on the insect family Drosophilidae [9]. Biologists examine the fruit fly, Drosophila, from the Drosophilidae family, to un-

derstand the basic principles of genetics [5]. Some of the basic principles they are looking for are the nature of genes, genetic linkage, meiotic chromosome segregation, signaling networks and recombination [5]. The signaling networks that researchers discovered in fruit flies are now recognized as central factors for major diseases, such as cancer, cardiovascular diseases, and neurological disorders [5]. Research on the Drosophila has also discovered major processes that affect humans, such as immune responses, stem cells, growth control, learning and memory, neural pathfinding, and synaptic transmission [5]. Drosophila is also used to model insect vectors of disease, such as malaria, dengue fever, yellow fever, and West Nile Fever [5]. Furthermore, the genus Drosophila has been used to understand population biology, the molecular basis of speciation, and evolution [5]. In short, the fruit fly is very important to biologists! Flybase seeks to be the primary database that stores the genetic information, publications, and 
terminology of the insect family Drosophilidae and allow collaboration among the fruit fly research community. Flybase is found online at http://flybase.org.

\subsubsection{Database back end}

The Flybase database stores practically all of the data in a single relational database using the Chado schema [11]. The Chado schema is used to store biological information from humans to pathogens [2]. The data is stored using ontologies. The ontologies are used as a means of typing entities. The schema then uses the types (ontologies) to create a graph that holds the relationships between these types. The schema is partitioned into subschemas; each subschema encapsulates a different biological domain and each is described using different ontologies [2]. For example, there is a subschema specifically for publications. Another is for just sequence data. More information on the Chado Schema is found in Appendix B.

\subsubsection{Tasks}

The Flybase website provides a variety of features for the user [10]. The functionality ranges from several implementations of bioinformatics algorithms that can be run on the data in the database, to viewing images of flies. A user can upload papers and genetic data via submission tool, and query the database using several different query tools. The website also includes tools to link pieces of data in the database to other data. Users can also download precomputed data directly from the websites FTP server. The data that can be downloaded includes a recent database dump, genes, alleles, ontology terms, genetic information, and more. Figure 3.1 shows one of the many query tools found on the Flybase website. 


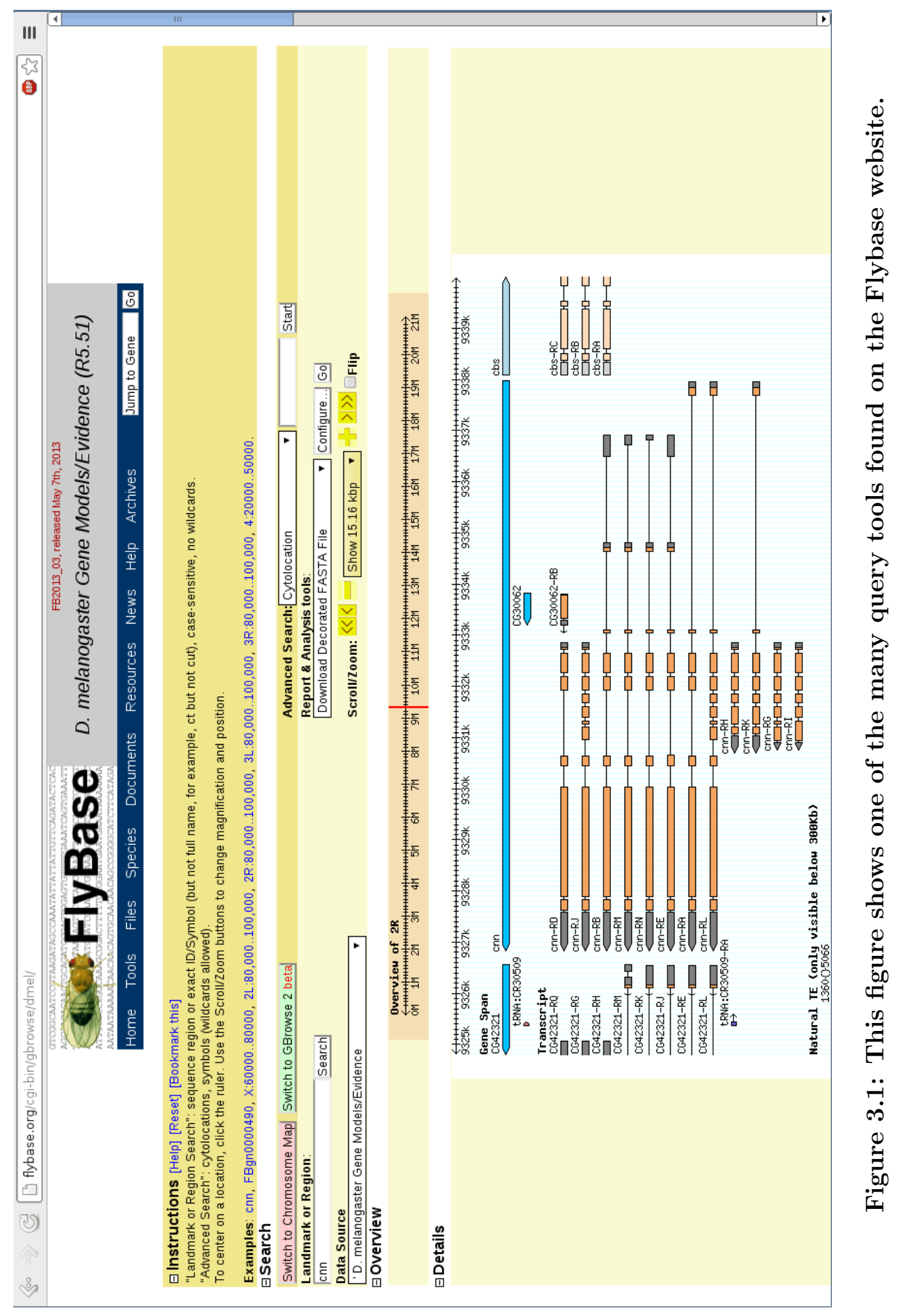




\subsubsection{Problems}

First of all it is important to examine what type of system the database is running on. The Chado schema is for relational databases so it is obvious that Flybase is running a relational database. Now depending on the configuration being used by the relational database, it can either be running a $\mathrm{CA}$ or $\mathrm{CP}$ system.

Next we need to examine the nature of data stored in Flybase. The data in Flybase is stored as graphs which are very similar to objects found in objectoriented programming. In order to store the data, several tables are used and to get the full graph, many joins must be used.

Not only is the data very object-oriented, the workload that is performed on Flybase does not justify having a $\mathrm{CA}$ or $\mathrm{CP}$ system. Updates and insertions are rare. The end-users of Flybase are querying from old data for around a month before a big update happens. Therefore, there is no need for the strong consistency guaranteed by the ACID transaction model. By switching to an AP distributed system, Flybase could scale more.

\subsection{Example 2: GenBank}

GenBank is a comprehensive genomics database that contains DNA sequences for around 260,000 species [19]. The DNA is obtained from submissions by individual laboratories and batch submissions from large-scale sequencing projects (like the whole-genome shotgun and environmental sampling projects). Via the NCBI Entrez retrieval system, the data from GenBank is incorporated with the major DNA and protein sequence databases along with taxonomy, genome, map- 
ping, protein structure and domain information, and the biomedical journal literature via PubMed.

\subsubsection{Database back end}

The back end for GenBank is a flat file database consisting of 1861 files with an uncompressed size of 594 GB [30]. For an example data file see appendix A.

The data format used in the flat files is called EST which is organized with keywords and their associated descriptions. Every line is considered a record, and the first 10 characters allow for the keyword or sub-keywords. The rest of the line contains the information pertaining to its keyword. There exist 18 different keywords that make up a possible EST entry. Different keywords define a specific meaning and usage. Some of the keywords are allowed to repeat in a given EST entry, such as the REFERENCE keyword.

Below is a quick guide to all of the keywords that could show up in an EST entry:

- The LOCUS field contains information on the GenBank record and a short name describing it. Information in the field includes how many base pairs are in the nucleotide snippet, what direction, type of sequence, ACCESSION number (described later), and the date submitted to GenBank.

- The DEFINITION field provides space for a definition of the sequence.

- The ACCESSION field has the record's ACCESSION number, as well as any ACCESSION numbers related to this record. Every record has a unique ACCESSION number for identifying purposes. 
- The VERSION field contains this record's ACCESSION number and a numeric version.

- The KEYWORDS field contains all annotated entries via a semicolon separated keywords list.

- The SOURCE field provides the common name of the organism that the DNA came from.

- The ORGANISIM field contains the formal scientific name of the SOURCE field along with other taxonomy information.

- The REFERENCE field allows the record to cite where it came from.

- The TITLE field is the full title of the citation from the REFERENCE field.

- The AUTHORS field lists the authors of the citation in the REFERENCE field.

- The CONSRTM field allows for a consortium of organizations to be authors rather than every individual in the organizations.

- The JOURNAL field has the journal name, page number, year, volume of the citation in the REFERENCE field.

- The REMARK field specifies the relevance of this citation in the REFERENCE field to this entry.

- The COMMENT field allows for additional comparisons, comments, and notes. 
- The FEATURES field has the biologically interesting information. For example, how the experiment was performed, where in the genome, and special information of portions of the sequence.

- The ORIGIN field has the point of origination in the genomic map and how the first base of DNA is reported. The ORIGIN field is followed by the DNA sequence data.

- The PUBMED field is used to reference the PubMed citation database [8].

- The MEDLINE field contains the Medline unique identifier if it exists for this record [7].

\subsubsection{Tasks}

The most common task performed is to display information via the NCBI Entrez retrieval system on a web page in human-readable form. The next most common tasks include daily updates of new sequences and providing major revisions of the database to researchers. These include a lot of updated data. A less common task is removing sequence data for reasons that could include privacy. Figure 3.2 shows the ACCESSION number CO069431 being retrieved via the NCBI Entrez system.

\subsubsection{Problems}

First, let us examine the system being used by GenBank. We know that GenBank uses a collection of flat files. We do not know if the files are stored on more than one server. Therefore, GenBank is either using a CA or CP system to run their flat file database. 


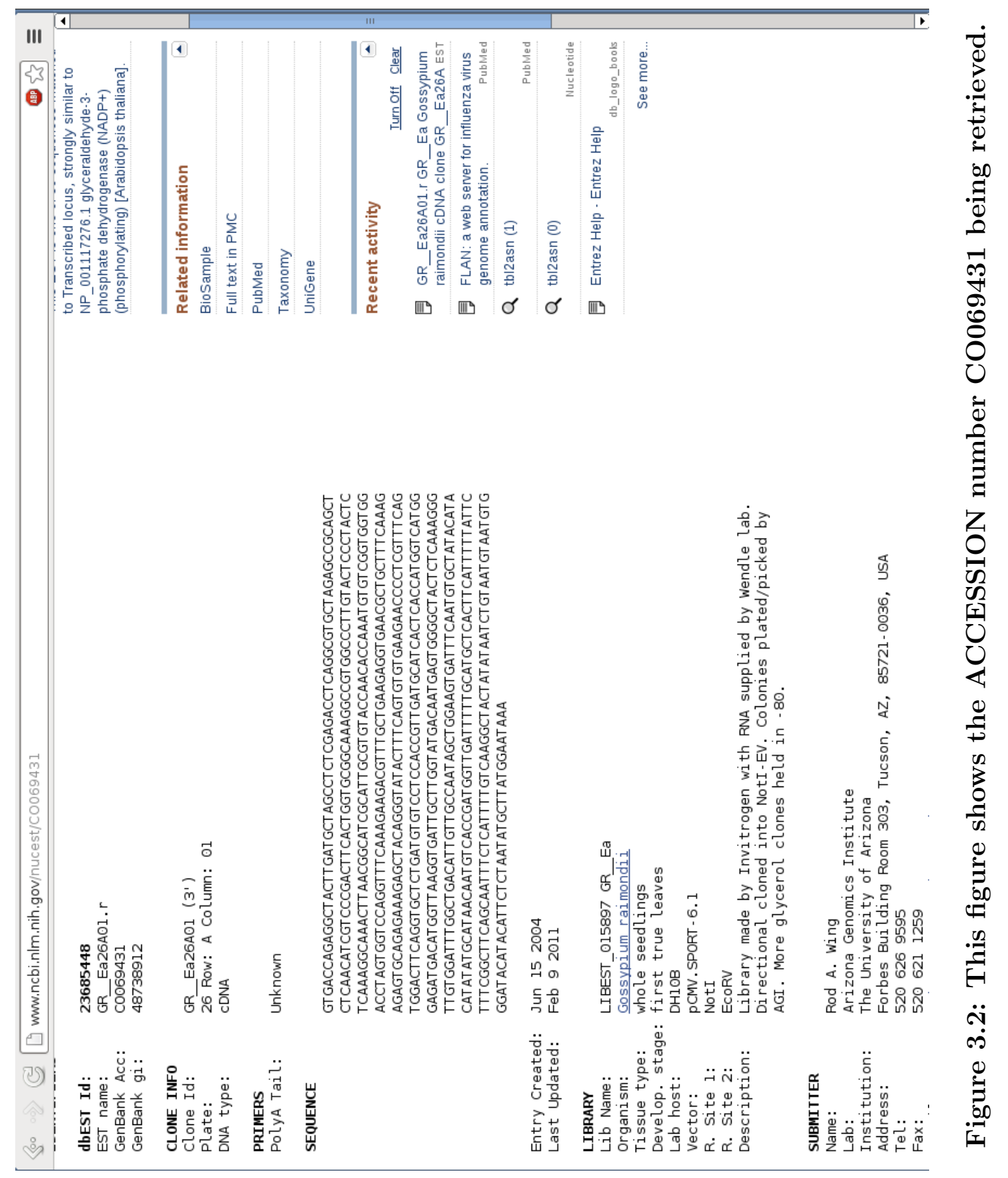


Next, let us examine the data being stored in GenBank. GenBank represents the ultimate key-value store. Records consist of all necessary data and have a unique identifier. Queries performed on GenBank are solely based on the unique identifier. So there is no need for any complex querying. Now that we know the data, let us examine the workloads performed by GenBank. Again, like in Flybase, most of the workload is querying the database for records and updates rarely happen.

From this I conclude that the database running GenBank does not need to provide consistency. By storing the data in a flat file CA system, all updates must go through GenBank and take up to 24 hours to show up. If GenBank was running an AP system, even with the eventual consistency, updates would not take even close to 24 hours to complete. Therefore, GenBank is a good example of a biological database that should be running an AP system.

\subsection{Conclusion}

From my research it should be clear that consistency is not needed in most biological databases. First, the workloads are read-heavy. So it would be rare that someone could read inconsistent data. Second, not only are the workloads readheavy but the queries performed are most often key/index based lookups. Both of these are what AP systems are designed for. Finally, the data and relationships between the data are complex and not easily represented in the commonly used $\mathrm{CA}$ and CP biological databases (flat files and relational databases). Often trees or graphs need to be used which is hard to represent and quickly query in flat files and in relational databases. 


\section{Chapter 4}

\section{Living without ACID}

So now that we have seen that AP systems are an excellent choice for biological database back ends, it is important to examine when AP systems make the most sense. From the previous chapter the following key principles should be present for an AP system to perform the best when dealing with biological data:

1. Read-heavy workload

2. No need for strict ACID transactions

3. Complex data/metadata

4. Querying is mostly key/index-based lookups

With these principles in mind, we needed to implement a biological database as a case study. Thus the Community Genome Annotation Training (CGAT) Database was born. 


\subsection{Community Genome Annotation Training ( $C G A T)$ Database}

Dr. Anya Goodman, a professor in the department of Chemistry and Biochemistry at Cal Poly, San Luis Obispo, offers a bioinformatics course that covers several aspects of gene annotation and genomic research. Her work is a part of the Genomics Education Partnership (GEP), based at Washington University in St. Louis [12]. GEP is an organization that aims to bring genomics research to academic institutions, especially undergraduate classes. This allows undergraduate students to get the opportunity to learn about, and practice, basic genomic research and, in turn, contribute meaningful work to the biological research community. However, GEP does not have an existing system in which users can input and save gene annotations and get immediate feedback regarding their performance. Similarly, available gene annotation software - RepeatMasker, Artemis, and others $[33,32]$ - is not designed to provide immediate feedback for users regarding their performance.

$C G A T$ has been built with usability, simplicity, and efficiency in mind. It is not a feature-rich and data-rich genome browser like UCSC genome browser [16], Ensembl [6], or BioViz [31], nor is it designed to be a replacement for gene annotation software such as RepeatMasker, Artemis, or any others, in any aspect other than for teaching, or practicing, gene annotation. Other gene browsers or annotation software offer many features to accommodate the needs of the biology community, and so tend to be complex. Gene annotation, while not a trivial task, can be well-serviced by a simple data model. Ideally, by focusing on a simple data model, $C G A T$ is able to provide a clean, streamlined experience capable of providing users with a way to view gene annotations made by others or have 
their gene annotations reviewed by experts. In particular, $C G A T$ strives towards a Wikipedia-like emphasis on collaboration and openness. CGAT stores a wide variety of data, including huge continuous stands of DNA, user data, collections of users, help pages with images, annotation data, and more. Each of the types of data are complex and require nested data-structures to fully describe them.

\subsection{Problem}

The following main use cases must be implemented.

1. People should be able to register and join $C G A T$.

2. Users should be able to upload contigs.

3. Users should be able to upload partial/finished annotations for a specific contig.

4. Users should be able to create and join groups.

5. Users should be able to create help pages.

6. Users should be able to get feedback after submitting annotations.

\subsection{Data}

$C G A T$ stores five crucial pieces of information: contigs, annotations, users, groups, and help pages. Contigs and annotations are biological in nature, while users, groups, and help pages are data that help manage $C G A T$. 
When biologists sequence genomes, they sequence them in overlapping segments called contigs. A contig, which is a DNA sequence of some length, contains some number of genes. Biologists record several pieces of information, called annotations, about genes including coordinates of components of the gene, such as exons, and meta information about the annotation like who uploaded it and when it was done, etc.

Groups are another important part of the usefulness of the system. Users of like minds or research interests can join the same group to receive important information. Users are a major part of the data and serve as the central hub that connects the data. Users upload contigs, create annotations, create help pages, and join groups. There exists the ability to create a help page for each page in $C G A T$. The help pages help describe and give tips about the page they refer to.

The following sections describe the major types of data found in $C G A T$ in more detail.

\subsubsection{Contigs}

Information stored about contigs includes:

- A large sequence of DNA nucleotides

- A name

- Name of the species the DNA came from

- A difficulty value

- Information on who uploaded the contig

- When the contig was uploaded 
- The status of the contig (test data, active, or practice)

- Map of isoform names to annotations for the given isoform

\subsubsection{Annotations}

Information stored about annotations includes:

- The isoform name of the gene being annotated

- The contig that was annotated

- Start and stop locations of the gene on the contig

- The exons of the gene being annotated

- Whether the annotation was submitted by an expert

- Whether the annotation was a partial submission or the complete submission

- Which strand of the DNA the gene was on (3'- 5' or 5'- 3')

- Who uploaded the annotation

- Metadata about the annotation - when it was created, submitted, and last modified

\subsubsection{Users}

Information stored about users includes:

- First name 
- Last name

- Login information - username, password hash and salt

- Email address

- The users role - default user, expert, admin

- Experienced gained from submitted annotations

- The users "level" - level up by gaining experience

- Metadata information - date they joined and last time they logged in

- Past annotation history

- Annotations that are currently in progress

\subsubsection{Groups}

Information stored about groups includes:

- The name of the group

- When the group was created

- The description of the group

- The list of users in the group

\subsubsection{Help pages}

Information stored about help pages includes: 
- The page that the document is for

- The title of the help page

- The HTML to be displayed on the help page

- When the help page was created

- When the help page was last modified

\subsection{Why are CA and CP systems a bad solution for $C G A T$ ?}

Now that we know more about the data being stored in CGAT, let us reexamine $\mathrm{CA}$ and $\mathrm{CP}$ systems to make sure they are not the best choice for a database back end.

The first thing to note is the wide variety of data being stored. Data ranges from long strands of DNA to HTML code. The next thing to remind ourselves about is the workload of CGAT. The vast majority of queries will be reads. Since the majority of the workload is reads, there is no need to ensure consistency on each node in the cluster. The last major thing to note is that transactions run in $C G A T$ are not transactional. Not transactional in the fact that if someone reads data while a transaction is running, nothing will be harmed. CGAT is not like a banking database where the last example is an extremely bad situation.

Let us also examine the most common choice for web-application database back ends and see that even relational databases have noticeable problems with the data stored in CGAT. Figure 4.1 depicts the ER-diagram for CGAT. From the ER-diagram it is easy to see that several issues arise quickly. Getting all of 
the data about an annotation requires three joins. As the amount of data in those tables increase, that query will be extremely slow. Other issues arise from the fact that sequences in contigs have to be stored as blobs. The size of sequences in contigs can vary from $10 \mathrm{k}$ nucleotides to $50 \mathrm{k}+$ nucleotides so there is no feasible option to use strings to store sequences without wasting lots of space. These are just some of the issues that would effect scaling with the SQL version of CGAT. Taking all of these issues into account, it is clear that the most common choice for a web-application is not meant for $C G A T$.

\subsection{Implementation}

CGAT uses a standard LAMP (Linux Apache MySQL PHP) stack with the ability to replace MySQL with any AP system. As long as it is possible for a database to implement the needed API calls, any system can be used. The implementation of CGAT started in CSC 560 in Fall 2012. We first implemented a Couchbase back end for CGAT because of how the course was structured and an assignment. After an evaluation comparing a MySQL back end for CGAT and our Couchbase version, we saw that Couchbase performed terrible for read-heavy workloads even though it was set up as an AP system.

Not being extremely fast for read-heavy workloads meant that Couchbase was a bad choice for CGAT. We examined why Couchbase was bad and saw that we needed more than a key-value store to perform better than MySQL. So we then created a MongoDB back end which is still being used in CGAT today. In what follows, we describe the MongoDB implementation. Then we go over how the Couchbase back end differed from the MongoDB implementation. 


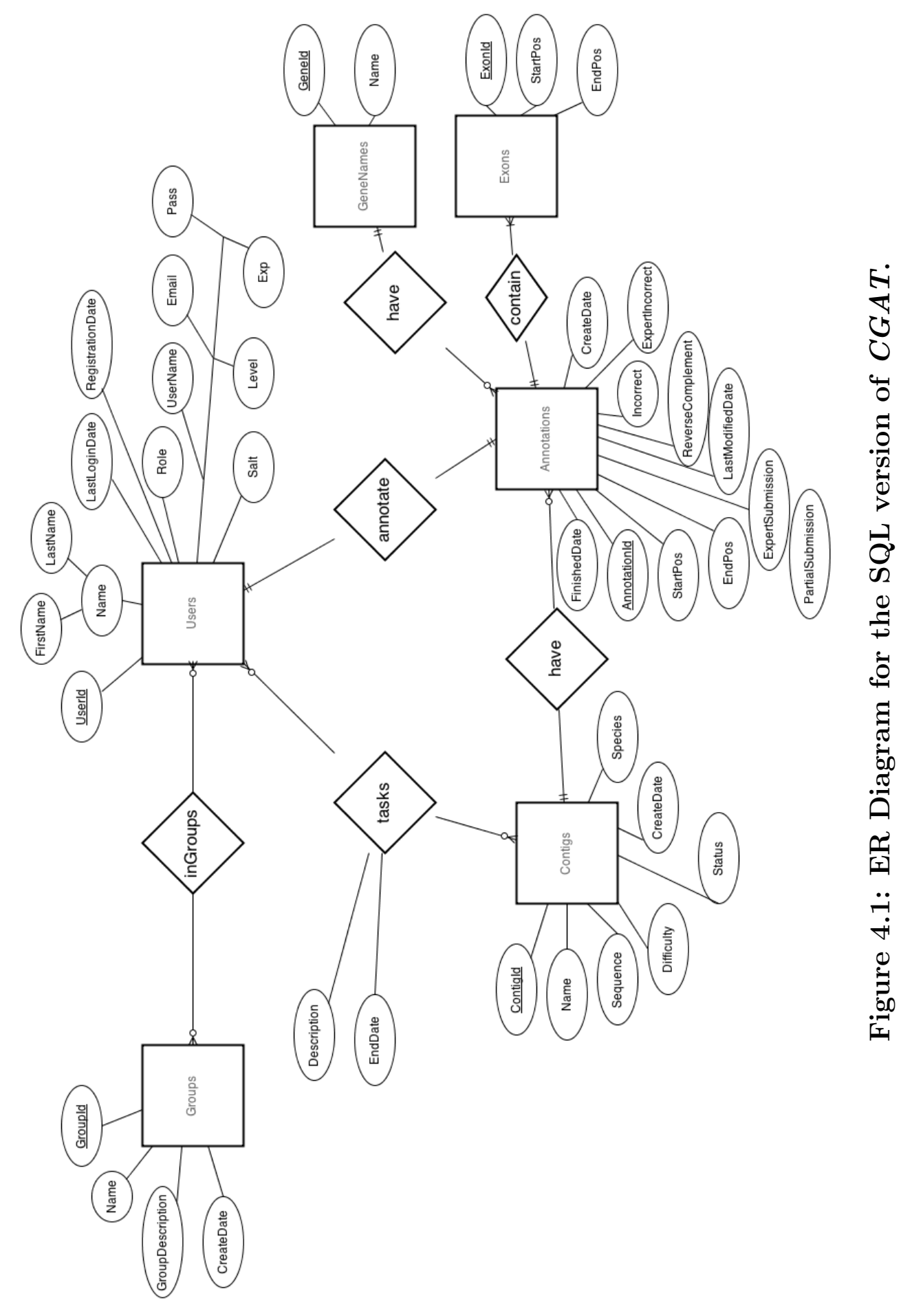




\subsubsection{Website Back End}

The website back end of the system consists of a PHP server connecting to a MongoDB database. We do not use any templating systems. We chose PHP for the back end because we did not require most of the functionality of a templating system, and we wanted a simple system that can be easily understood when passed off to the next generation.

PHP and MongoDB work together very nicely and MongoDB's PHP connector is fully featured [27].

\section{Database Schema}

Although we use MongoDB, which does not enforce a specific schema, by convention we enforce a schema on our documents. MongoDB uses BSON (Binary JSON). BSON is an extended JSON format that supports additional types like a datetimes [26]. MongoDB stores documents in collections. We had a separate collection for each type of document. Figures 4.2, 4.3, 4.4, 4.5, 4.6 depict the BSON version of each type of document found in CGAT. 


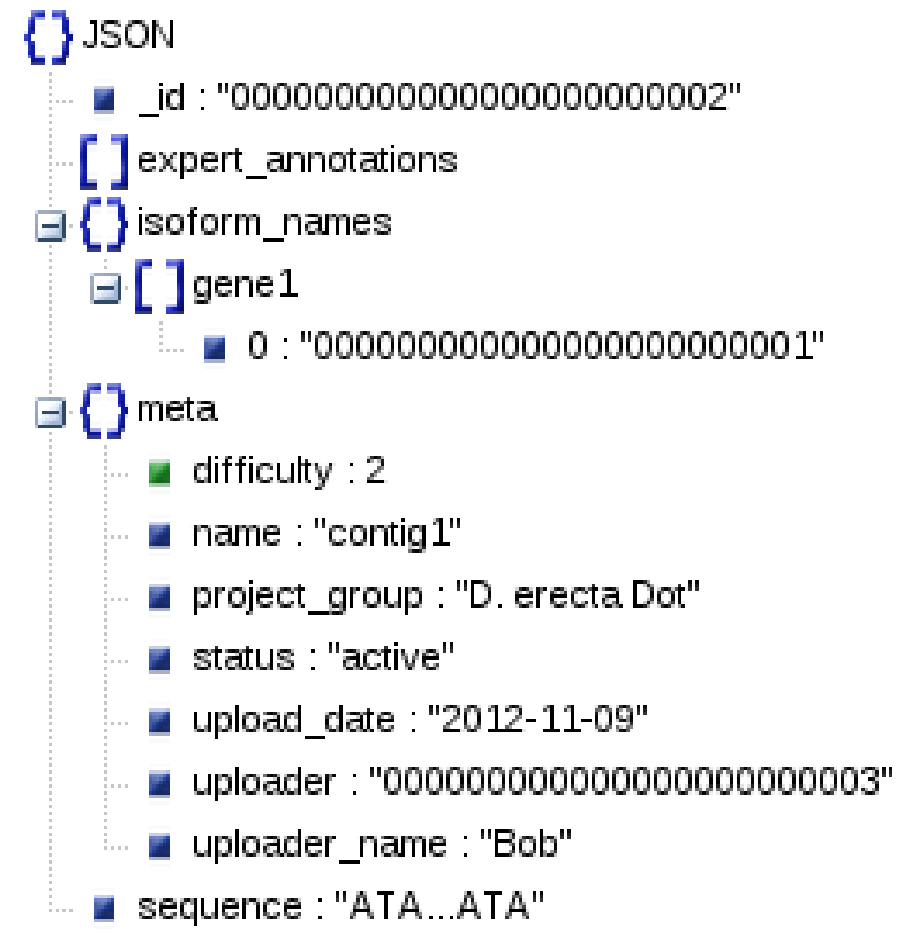

Figure 4.2: Example contig document.

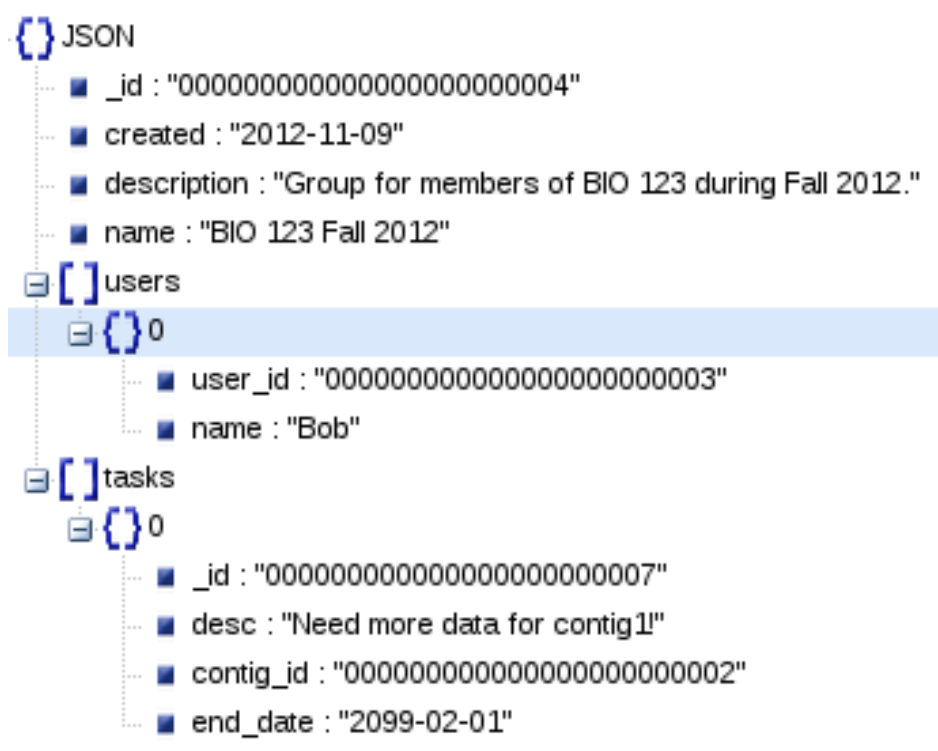

Figure 4.3: Example group document. 


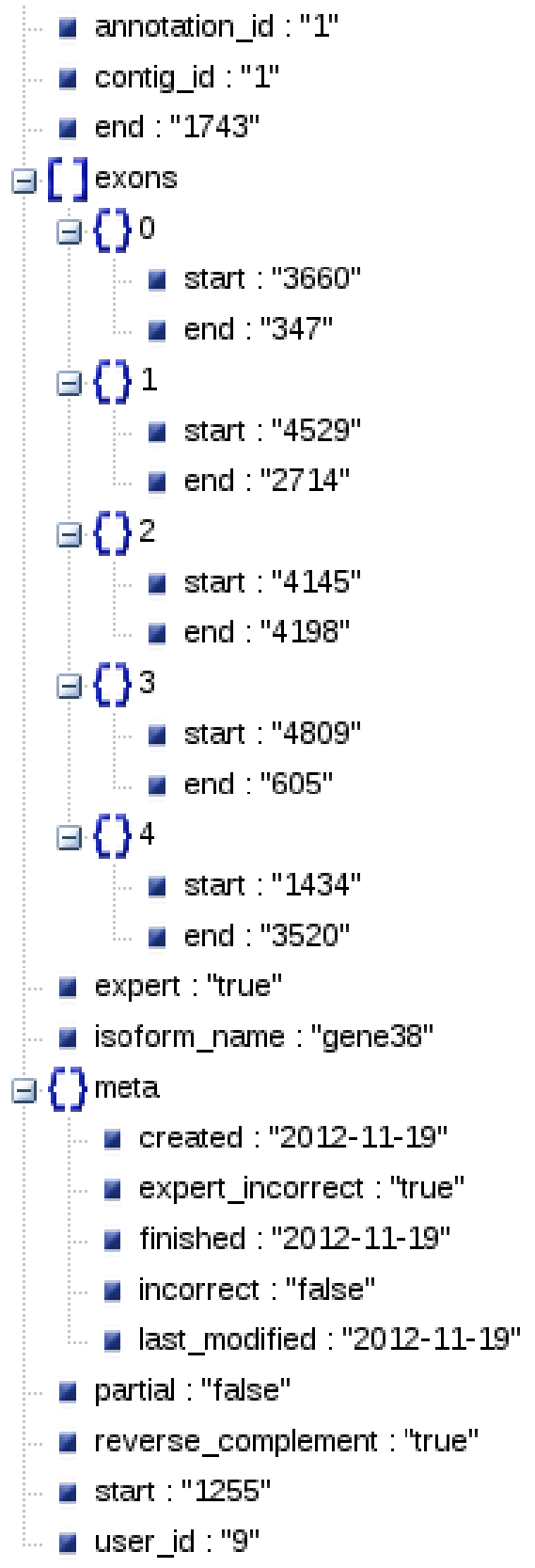

Figure 4.4: Example annotation document. 


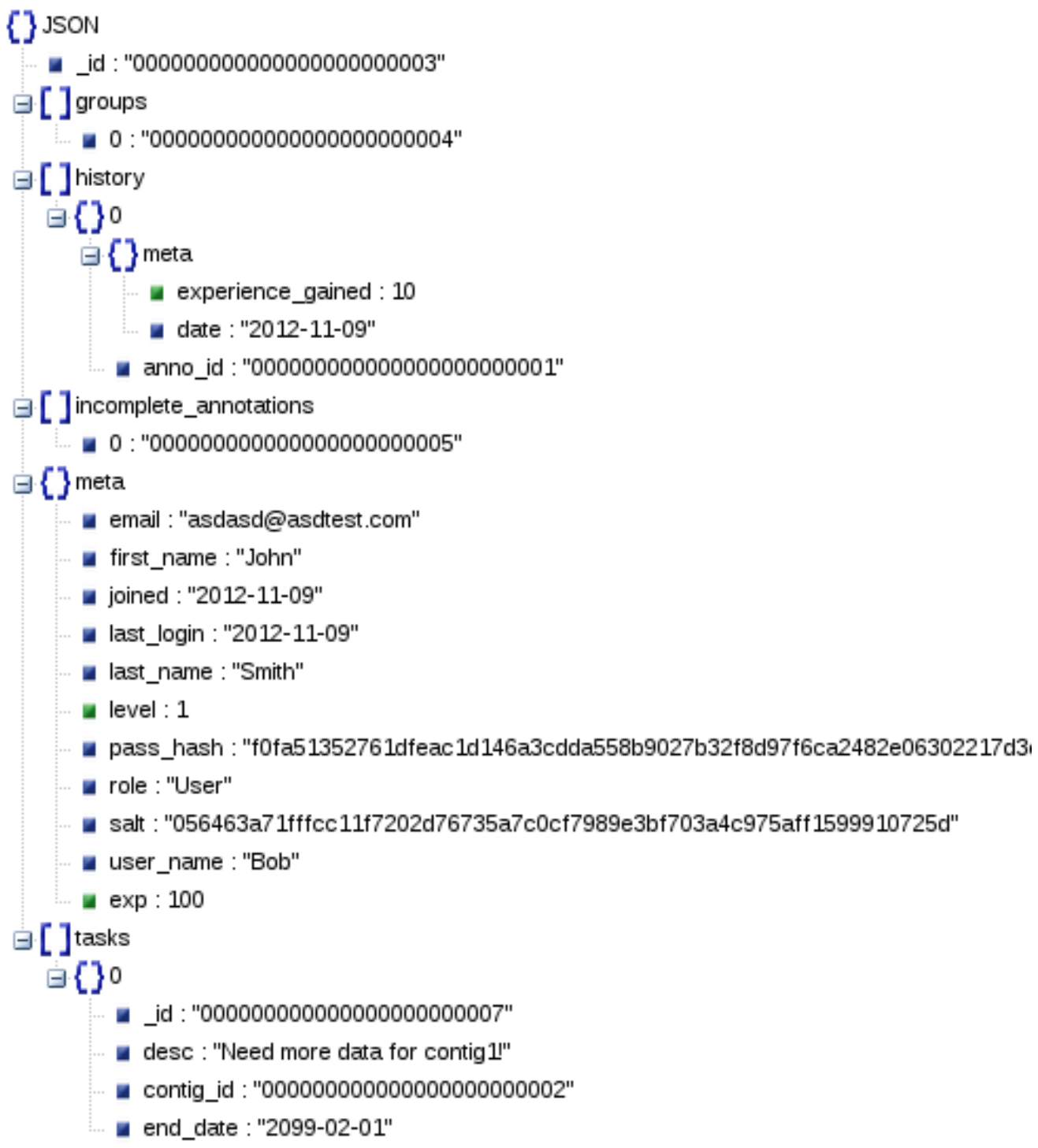

Figure 4.5: Example user document.

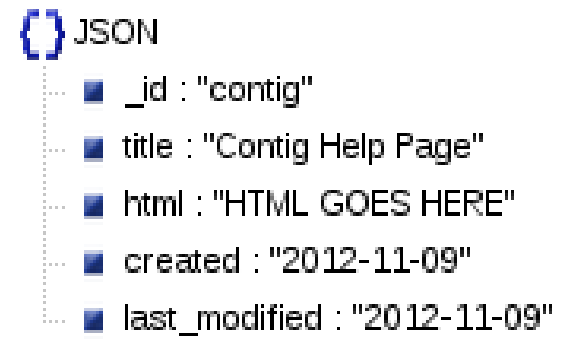

Figure 4.6: Example help page document. 


\begin{abstract}
API
$C G A T$ is an entirely API-driven product. The API is central to all the behavior. The API supplies all the information that is displayed in the UI. This is a very important design choice. Having the API be the sole data provider and manipulator for the system means that all functionality can be programmatically provided and different applications can present the same functionality with a different UI. Thus the programmers working at GEP can extend current genome browsers to store data in $C G A T$.

The API accepts GET and PUT requests (depending on the action) and will always return a JSON response. Having an API is why switching out the database back end is extremely easy. As long as any database provides a PHP driver, it can be used in CGAT. To switch out the database, all that needs to happen is to implement the API calls in the new database,s PHP driver. The current API supports 23 different calls, 9 GET requests, and 14 POST actions.
\end{abstract}

GET Requests. Most get requests do not require the user to be logged in. Table 4.1 lists the get requests found in the CGAT API. 


\begin{tabular}{|l|l|}
\hline Get Request Name & Information \\
\hline administration_info & $\begin{array}{l}\text { Provides the user with meta information that the user } \\
\text { would need to perform administrative tasks such as cre- } \\
\text { ating a group or assigning a task. }\end{array}$ \\
\hline annotation & $\begin{array}{l}\text { Provide the user with information about a specified an- } \\
\text { notation. }\end{array}$ \\
\hline contig & Get information about a specific contig. \\
\hline claim & $\begin{array}{l}\text { Get all contig information in a specific project group. } \\
\text { Used for claiming new contigs to annotate. }\end{array}$ \\
\hline gene & Get information about a specific gene. \\
\hline get_feedback & Get feedback for a specific annotation. \\
\hline group & Get information about a specific group. \\
\hline help & Get help information on a specific topic. \\
\hline user_profile & $\begin{array}{l}\text { Get the profile information about a specific user. If the } \\
\text { user is logged in and requests information about them- } \\
\text { selves, more information is provided. }\end{array}$ \\
\hline
\end{tabular}

Table 4.1: Table of the get requests found in the $C G A T$ API.

POST Actions. Most POST actions require that the user is logged in. Table 4.2 lists the post requests found in the CGAT API. 


\begin{tabular}{|l|l|}
\hline Post Request Name & Information \\
\hline assign_task & Ask a group of people to perform an annotation. \\
\hline cancel_notification & Clear a notification from a user. \\
\hline create_annotation & Create a new annotation of a contig. \\
\hline create_group & Create a group. \\
\hline join_group & Join a group. \\
\hline leave_group & Leave a group. \\
\hline login & Login. \\
\hline logout & Logout. \\
\hline parse_fasta & $\begin{array}{l}\text { Upload a FASTA file to the server, parse the contents, } \\
\text { and return the parsed structure. }\end{array}$ \\
\hline register & Register a new user. \\
\hline save_annotation & Save a work-in-progress annotation. \\
\hline set_help & Upload a help page. \\
\hline submit_annotation & Submit a finalized annotation. \\
\hline upload_contig & Upload a contig into CGAT. \\
\hline
\end{tabular}

Table 4.2: Table of the post requests found in the $C G A T$ API.

\subsubsection{Front End}

CGAT's front end uses standard web technologies (HTML, Javascript, and CSS). CGAT uses many HTML5/CSS3 features such as transforms and pseudoelements. Because the entire system is API based, the front end heavily relies on asynchronous fetches (AJAX).

\subsubsection{Common Workloads}

This section contains the most common workloads run on CGAT. By far the most common are the first three workloads which are read-heavy. Each workload is discussed below in turn. 


\section{Profile View}

The first thing that all users will do in $C G A T$ (ideally) is log in. When a user logs into $C G A T$, the user is directed to their profile view which contains all groups they are a member of, all completed annotations they have submitted, partial annotations that have been saved, and all tasks they have assigned. This is a workload that is likely to see many additions in the future as it serves as the first and foremost view of the system for the user. This means that any information that should be accessible - including when a forum system is integrated into $C G A T$ - will find a place on the user's profile.

This workload is clearly a read-heavy workload that entails no disk writes at all. Important to note though, this use case may constantly be increasing (should constantly be increasing for a successful system) and so the may increase quite a bit, and whenever new features are added, there is a potential for a spike in workload.

\section{Task Assignment}

In $C G A T$ it must be possible to request a group of users to annotate new or updated contigs. The frequency of this use case is dependent on the adoption of $C G A T$ - the more species $C G A T$ supports, the more frequent this use case will be. More specifically, the relevant entities in the CGAT schema are users, contigs, and groups. Since a task embodies a request for a group $G$ to annotate a contig $C$, every user $U$ in $G$ receives a notification including a description of $C$ and a message requesting that it be annotated. A task can be assigned to

any number of groups. Since tasks are assigned to groups that have some vested interest in the species or specific contig that has been updated or added, there 
may be many groups that a task should be assigned to. Additionally, as more species are supported by $C G A T$ or more users join $C G A T$, more users will receive notifications of tasks and so there are multiple ways in which this workload must be scalable.

This workload is read-heavy since group, contigs, and users must be queried before adding tasks.

\section{Annotation Publication}

Annotations are the core focus of $C G A T$. Every user is going to be uploading some annotations. Hopefully they should be uploading many annotations. Especially since $C G A T$ defines an annotation to be information about a gene isoform, variation, on a contig, even annotating a single gene will yield several annotations in $C G A T$. Additionally, when an annotation is submitted, the annotation is marked as complete, the user and annotation are then assigned a certain amount of experience - the user gains experience towards his or her total, the annotation gains experience and serves as a history of the user's experience.

This is a mostly read-heavy workload with some writes. It is mostly readheavy in the sense that it must eventually do some writing by updating a user and the annotation that the user completed. However, it also has a lot of reads in the sense that the contig, for which the annotation is being submitted, and the

user have to be queried to determine the amount of experience that submitting the annotation yields for the user. 


\section{Group Modification}

Group membership is likely the second most stable of the workloads. This is "stable" because the majority of users will only join groups, and leaving groups will likely be relatively rare. This workload will only ever be as bad as how many users join at any one time - once a user registers for CGAT and has joined some groups, the user is unlikely to join or leave groups, and so this workload poses almost no scalability problems. Although, despite this, there will be spikes that coincide with academic schedules, as groups will be created and populated for each new class that uses $C G A T$.

This workload is the most balanced of the workloads. Generally speaking, when a user joins a group, then there is only the addition of a user id in the group document and a group id in the user's document. And when a user leaves a group, then the previous changes are undone by removing the user id and group id from their respective documents.

\section{Contig Uploads}

Contig uploads is the most stable workload when the manual, or user, use case is considered. Although, there will be large spikes of activity when/if the reference genome for a species is updated,requiring $C G A T$ to update any and all of the contigs relevant to the genome. However, reference genomes are updated very infrequently and contigs will never be removed from $C G A T$.

This workload is write-heavy because it requires no reads - the workload is for uploading data, not downloading data. 


\subsubsection{Couchbase Implementation}

The Couchbase back end slightly differed from the MongoDB version. The Couchbase implementation stored the same JSON documents as the MongoDB implementation. The only difference was how documents were accessed. In Couchbase a user document for the user with the ID of 1 was stored with the key "users-1". Same idea for the group document with the ID of 5 ("groups-5"). Whenever a document that was being manipulated had a reference to another

document, the way to query for the referenced document was by hyphenating the type of document with the ID being searched for. 


\section{Chapter 5}

\section{Evaluation}

Traditional web applications are almost always backed by a relational database. Since $C G A T$ is being backed by an AP system it is important to consider the performance ramifications. The main goal of the evaluation is to determine how an AP implementation compares to a traditional back end like MySQL. If my argument is correct, the AP implementations should perform better for read-heavy workloads. For the evaluation we compared a CP configuration of MySQL, an AP configuration of Couchbase and an AP configuration of MongoDB.

\subsection{Experiment Design}

To evaluate the performance of the different implementations, we generated around $400 \mathrm{MB}$ of data that was used by each system ${ }^{1}$. We then created a set of six experiments to run. Five came from common workloads found in CGAT. Recall from Section 4.5.3 that these are profile view, publishing annotations, group modification, contig upload, and task assignment. The final experiment was a

\footnotetext{
${ }^{1}$ The data models used are depicted in Chapter 4.
} 
contrived one designed to test the performance on each cluster by repeatedly writing and reading from the same object. Our major metrics are write performance, read performance, and time-delay between writes and reads of the same data.

For our experiments, we are using a total of five EC2 instances from the Amazon Web Services cloud offering. For all of the implementations, four servers were used for the database cluster while the fifth was used for running our workload harness. The MySQL system is setup with one master server for writes and three slave servers that replicate all of the masters data. Similarly, the MongoDB system was setup with one master for writes and three replicas for read performance. The Couchbase system was configured in a way where each server handled a subset of the data. The main tasks of the workload harness was to define how many times a workload would run and which workloads to run. In our case, workloads represent a certain task or use case in $C G A T$. For example, retrieving data that is displayed on a user's profile. In our system, a workload knows how to do its job in all of the implementations. As each workload is run, the workload harness records important statistics. These include the length of time the workload took to complete, the min and max completion times of an individual query or insertion, and the standard deviations of individual runs.

\subsubsection{Data}

Our data models have four crucial pieces of information: contigs, annotations, users, and groups. We chose to not use help pages in our experiments because they did not show up in the common workloads. 


\begin{tabular}{|l|c|c|c|}
\hline Workload & MySQL & Couchbase & MongoDB \\
\hline Task Assignment & $39325189 \mathrm{~ms}$ & $157431049 \mathrm{~ms}^{2}$ & $5327456 \mathrm{~ms}$ \\
\hline Profile View & $2908257 \mathrm{~ms}$ & $7008818 \mathrm{~ms}$ & $165409 \mathrm{~ms}$ \\
\hline Annotation Publication & $510837 \mathrm{~ms}$ & $1830064 \mathrm{~ms}$ & $324601 \mathrm{~ms}$ \\
\hline Group Modification & $316673 \mathrm{~ms}$ & $9086304 \mathrm{~ms}$ & $1322606 \mathrm{~ms}$ \\
\hline Contig Upload & $119892 \mathrm{~ms}$ & $20133 \mathrm{~ms}$ & $57124 \mathrm{~ms}$ \\
\hline Read/Write & $103808 \mathrm{~ms}$ & $58581 \mathrm{~ms}$ & $152268 \mathrm{~ms}$ \\
\hline
\end{tabular}

Table 5.1: Comparison of runtimes in $\mathrm{ms}$ for the different workloads. Each workload was run for 100000 iterations.

\section{MySQL}

Our data model in SQL consists of eight relational tables. An ER-diagram of the tables is depicted in Figure 4.1 from Section 4.4. In the tests we also added an additional table for the last experiment that tests reading and writing from the same object. The CREATE TABLE statements are located in Appendix C.

\section{Couchbase and MongoDB}

All of the data in Couchbase and MongoDB is represented in JSON. We used four JSON objects which are shown in Figures 4.2, 4.4, 4.5, and 4.3 in Section 4.5. All of same information from the MySQL tables is represented in these JSON objects. We take advantage of nested objects to reduce as much subsequent requerying of the database as possible.

\subsection{Workloads}

The core unit of work that we used to compare performance was called a workload. Unfortunately, due to time constraints we were unable to do multiple

\footnotetext{
${ }^{2}$ Task assignment never actually completed. The number reported is the approximate length of time spent running the workload before giving up. Explanation provided in Section 5.4
} 


\begin{tabular}{|l|c|c|c|}
\hline \multicolumn{4}{|c|}{ MySQL read/write performance } \\
\hline & RAW & WAR & combined \\
\hline Min (ms) & 0 & 0 & 0 \\
\hline Max (ms) & 25 & 70 & 70 \\
\hline Mean (ms) & 0.473 & 0.558 & 0.516 \\
\hline Median (ms) & 0 & 1 & 0 \\
\hline Standard Deviation (ms) & 0.685 & 0.859 & 0.778 \\
\hline
\end{tabular}

Table 5.2: Comparison of reads and writes to the same object in MySQL.

runs of any one workload, however, due to our environment and machines, there should be minimal fluctuations in any given run time for a workload.

The workloads we are running include the five most common workloads in $C G A T$. Recall from Section 4.5.3 that these are profile view, publishing annotations, group modification, contig upload, and task assignment. The last workload we ran is discussed below.

\subsubsection{Read/Write Performance}

This is a test workload to examine the performance of writing and reading from the same object in succession. This is a naive, balanced workload with even numbers of read and writes.

\subsection{Implementation}

Our experiment was implemented in Java. To do the MySQL portion of the workload, we used the MySQL JDBC driver. For the Couchbase portion we used the official Couchbase Java library from their website. Finally, for the MongoDB portion we used the official MongoDB Java driver. 


\begin{tabular}{|l|c|c|c|}
\hline \multicolumn{4}{|c|}{ Couchbase read/write performance } \\
\hline & RAW & WAR & combined \\
\hline Min (ms) & 0 & 0 & 0 \\
\hline Max (ms) & 71 & 48 & 71 \\
\hline Mean (ms) & 0.562 & 0.017 & 0.290 \\
\hline Median (ms) & 1 & 0 & 0 \\
\hline Standard Deviation (ms) & 0.859 & 0.213 & 0.682 \\
\hline
\end{tabular}

Table 5.3: Comparison of reads and writes to the same object in Couchbase

\begin{tabular}{|l|c|c|c|}
\hline \multicolumn{4}{|c|}{ MongoDB read/write performance } \\
\hline & RAW & WAR & combined \\
\hline Min (ms) & 1 & 0 & 0 \\
\hline Max (ms) & 11 & 1 & 11 \\
\hline Mean (ms) & 1.491 & 0.027 & 0.759 \\
\hline Median (ms) & 1 & 0 & 1 \\
\hline Standard Deviation (ms) & 0.514 & 0.162 & 0.826 \\
\hline
\end{tabular}

Table 5.4: Comparison of reads and writes to the same object in MongoDB.

\subsection{Evaluation}

In this section we discuss the results for each workload in turn. As a general note, each workload was ran $N$ number of times. While we initially planned for $N$ to be the same for each workload $(N=100000)$, the ContigUpload workload ran into memory constraints of the EC2 machine running our queries. This caused us to reduce the number of times the task was repeated $(N=10000)$ for the ContigUpload workload. Additionally, the TaskAssignment workload never completed on Couchbase. The runtime reported for this workload was the amount of time the TaskAssignment workload was running. We had waited approximately 45 hours before finally killing the workload harness. 


\subsubsection{Task Assignment}

From Table 5.1, it is obvious that MongoDB significantly outperforms both MySQL and Couchbase for the TaskAssignment workload. We think this is due to the read-heavy nature of this workload. MySQL performed better than Couchbase because MySQL's data model allows simple ad-hoc query to find necessary values prior to inserting the new task, unlike Couchbase which must fetch entire JSON documents from the database and it must do this for several documents for each task being assigned. Since this workload only requires a small subset of the JSON documents to be used, MongoDB was able to take full advantage of its document oriented querying capabilities.

\subsubsection{Profile View}

Similarly to the TaskAssignment workload, MongoDB vastly outperforms MySQL and Couchbase for the ProfileView workload. Likely, this is for the same reasons that the TaskAssignment workload has poor performance on Couchbase and the fact the MySQL has to do many joins to retrieve a given user's profile.

\subsubsection{Annotation Publication}

Again, a similar pattern in the results as the last two workloads. This was another workload that required several queries prior to inserting a new annotation. MongoDB was able to leverage querying parts of documents to avoid having to retrieve necessary data. Whereas Couchbase had to retrieve the entire sequence of every contig. 


\subsubsection{Group Modification}

To our surprise this workload performed extremely poorly on Couchbase. We were not expecting this because this is a $50 \%$ read and $50 \%$ write workload. Having to do lots of reads prior to inserting new group memberships really hurt performance for Couchbase. It seems that Couchbase simply does not lend itself well to highly related or inter-connected data. Since there were so many writes MongoDB did not perform as well as MySQL.

\subsubsection{Contig Uploads}

The results from this test were no surprise, the ContigUpload workload is entirely write-heavy and so Couchbase performance demolishes the MySQL and MongoDB performance. Couchbase is optimized for a lot of writes. Since Couchbase does not have to lock key-value pairs, Couchbase can achieve great write performance. MongoDB also performed better than MySQL which is no surprise because it too does not have to lock the database on writes.

\subsubsection{Read/Write Performance}

The results from this test were surprising. MongoDB performs poorly when it has to read a document that has just been written too. Which ended up hurting its overall runtime. Couchbase performed the best because anything with writes it finished extremely quickly. For this workload, we decided to gather more statistics to see how the database performs. We expanded our statistics gathering to include the minimum, maximum, mean, median and standard deviation of the individual reads and writes for both Couchbase ,MySQL and MongoDB. Table 5.2 contains 
our SQL results, while Table 5.3 contains equivalent results for Couchbase and Table 5.4 contains equivalent results for MongoDB. All tables list the minimum, maximum, mean, median, and standard deviation for various types of database access patterns. The types of database access patterns we were interested in are reads after writes of the same object and writes after reads of the same object. Examining the read after writes results from the three systems, we observe that MongoDB has the worst performance reading after writing. Both Couchbase and MongoDB have better performance than MySQL when it comes to writing after reading.

\subsection{Conclusions}

From our results, MongoDB performs amazingly well on the read-heavy workloads. This backs up my argument that biologists should switch to an AP system, especially if most of the workloads are read heavy. It is clear that the document oriented nature of MongoDB helped it outperform Couchbase. So it may be worth performing further tests to evaluate whether only document store AP systems are best for biological data or if Couchbase just did not work well. MySQL appears to provide better performance for the balanced workloads. Whereas, Couchbase performs the best for write-heavy workloads. In the end, it's possible that Couchbase may not perform as badly if we could optimize for it. The amount of effort required to achieve our current performance statistics was much less for both MySQL and MongoDB. 


\section{Chapter 6}

\section{Conclusions and Future Work}

\subsection{Conclusions}

In conclusion, this work presented an argument that the current way of storing biological data is catastrophically inefficient. Continually adding new specialized databases will not solve the problem of increasing amounts of data. There needs to be a fundamental change in the way the data is stored. We argue that the nature of the workloads run on biological databases, the complexity of the data, and the fact that the transactions done in the biological databases do not need the ACID constraints, lead to AP systems as the best choice for biological database back ends. As a case study, CGAT was implemented with an AP system back end to show the benefits of an AP database solution. CGAT met the initial round of use cases created by Dr. Anya Goodman. An evaluation was done which compared the AP implementations of $C G A T$ to the $\mathrm{CP}$ version. It was clear that for the workloads expected of $C G A T$, that MongoDB was an excellent back end. Which is what my argument predicted. From the results, it was also clear that Couchbase as an AP system performed abysmally for the important workloads. 
Therefore, further work should be done to figure out the best type of AP system to use for biological data. There are a variety of options to evaluate. The options include document store, key-value, and column-oriented types of databases.

\subsection{Future Work on $C G A T$}

The first version of $C G A T$ is now complete. Yet there is still more to be done. The following list contains new major features to be implemented in CGAT.

- Forum - For every group, contig, and gene there should be a forum associated with it.

- Collaborative Annotations - There needs to be a method for clustering all annotations from the community and experts into a collaborative or representative annotation. This is not an easy problem. Things to consider include: if previous user submissions count as much as the latest submission, if experts' annotations weigh more than normal user's, if more recent submissions are weighted higher, etc.

- Review or challenging capability - Users or other experts must be able to review annotations by other experts or users and mark them as incorrect or wrong. Original author of the annotation must be able to respond to the review/challenge. Need a mediating process to examine the disagreement. A good model for this type of mediation are the ticket systems used to manage software bugs in large projects.

- Hints - Hints must be added to contigs so that when a user repeatedly submits an annotation for a gene, they will get hints if they are struggling. 
- Update annotation page - Allow uploading and parsing of GFF files and exon ranges.

- Leveling - Determine how to award experience and how levels are going to work.

- Removing expired tasks - Either check/remove old tasks on every access or via a daily cron job.

- Practice contigs - The correct annotation is known at the time of uploading. Should be able to upload a GFF file with the correct annotation at the same time as the contig is uploaded.

- Flybase version with every contig - Need to save the current Flybase version on contig uploads.

- Verify that submitted gene names are real - Use Wilson's GEP code to verify that the gene-isoform name is legit.

- Verify that contigs being uploaded are not already in $C G A T$ - Maybe use contig name, the range, and project group as a unique identifier.

- Administration page for user management - Create a user administration page for signing user roles and resetting passwords.

- Deleting/Marking annotations - Deleting or being able to mark your own annotations as wrong so they are not included in the collaborative annotation.

- Accessibility testing - Several of the color schemes might not work well with colorblind users. 
- Extend the claim page with more search options (contig difficulty, upload date, etc).

- Finish implementing the contig page - Need to display all known genes that have been annotated from the contig.

- Finish implementing the gene page - Need to display the collaborative annotation or expert/reference annotations for the gene.

- Finish implementing the search page - Need to be able to search for contigs, genes, users, groups, and annotations.

- Add a stats page - The page would display a given user's attempted contigs, number of annotations, number of genes attempted, number of challenges done, level, history, etc.

The following list contains the known bugs that must be fixed in CGAT.

- When submitting an expert annotation, the annotation ID is not being added to the correct contig document's expert_annotations field.

- Older versions of IE do not work with CGAT. 


\section{Bibliography}

[1] Bioinformatics factsheet. http://www.ncbi.nlm.nih.gov/About/primer/bioinformatics.html.

[2] A chado case study: an ontology-based modular schema for representing genome-associated biological information. http://bioinformatics.oxfordjournals.org/content/23/13/i337.

[3] Couchbase Server Features. http://www.couchbase.com/couchbaseserver/features.

[4] Domain name systems. http://en.wikipedia.org/wiki/Domain_Name_System.

[5] Drosophila board white paper 2009: prepared by the board. http://flybase.org/static_pages/news/whitepapers/DrosBoardWP2009.pdf.

[6] Ensembl genome browser home. http://uswest.ensembl.org/index.html.

[7] Fact sheet: Medline. http://www.nlm.nih.gov/pubs/factsheets/medline.html.

[8] Fact sheet: Pubmed: MEDLINE retrieval on the World Wide Web. http://www.nlm.nih.gov/pubs/factsheets/pubmed.html.

[9] FlyBase: enhancing drosophila gene ontology annotations. http://nar.oxfordjournals.org/content/37/suppl_1/D555.long.

[10] FlyBase Homepage. http://flybase.org/. 
[11] FlyBase: integration and improvements to query tools. http://nar.oxfordjournals.org/content/36/suppl_1/D588.long.

[12] Genomics Education Partnership - GEP Home Page. http://gep.wustl.edu/.

[13] JSON. http://en.wikipedia.org/wiki/JSON.

[14] MongoDB. http://www.mongodb.org/.

[15] Transcript of the interview with Dr. Atul Butte - multimedia - National Institute of General Medical Science. http://publications.nigms.nih.gov/multimedia/butte_transcript.html.

[16] Ucsc genome browser home. http://genome.ucsc.edu/.

[17] Visual guide to NoSQL systems. http://blog.nahurst.com/visual-guide-tonosql-systems.

[18] Database definition. http://en.wikipedia.org/wiki/Database, Apr. 2013.

[19] D. A. Benson, I. Karsch-Mizrachi, K. Clark, D. J. Lipman, J. Ostell, and E. W. Sayers. GenBank. Nucleic acids research, 40(D1):D48-D53, 2012.

[20] A. Birkland. BIOZON: a hub of heterogeneous biological data. Nucleic Acids Research, 34:D235-D242, Jan. 2006.

[21] E. Brewer. Towards robust distributed systems. http://www.eecs.berkeley.edu/ brewer/cs262b-2004/PODC-keynote.pdf, July 2000.

[22] F. Bry and P. Kröger. A computational biology database digest. http://dl.acm.org/citation.cfm?id=607994, 2003. 
[23] D. Emmert. The new and improved chado genetics module. http://gmod.org/mediawiki/images/5/5c/Emmert_040526.pdf.

[24] M. Y. Galperin. The molecular biology database collection: 2008 update. Nucleic Acids Research, 36(Database issue):D2-D4, Jan. 2008. PMID: 18025043 PMCID: 2238887.

[25] S. Gilbert and N. Lynch. Brewer's conjecture and the feasibility of consistent, available, partition-tolerant web services. lpd.epfl.ch/sgilbert/pubs/BrewersConjecture-SigAct.pdf.

[26] T. P. Group. Bson - binary json. http://bsonspec.org.

[27] T. P. Group. Mongodb native driver. http://us2.php.net/mongo, Nov. 2012.

[28] T. Haerder and A. Reuter. Principles of transaction-oriented database recovery. ACM Computing Surveys, 15:287-317, Dec. 1983.

[29] W. C. L. III, J. M. Williams, M. E. Mangan, and M. E. Mangan. Genomic Data Resources: Challenges and Promises. http://www.nature.com/scitable/topicpage/genomic-data-resourceschallenges-and-promises-743721.

[30] NCBI. NCBI-genbank flat file release 195.0. ftp://ftp.ncbi.nih.gov/genbank/gbrel.txt.

[31] J. W. Nicol, G. A. Helt, S. G. Blanchard, A. Raja, and A. E. Loraine. The integrated genome browser: free software for distribution and exploration of genome-scale datasets. Bioinformatics, 25(20):2730-2731, 2009.

[32] K. Rutherford, J. Parkhill, J. Crook, T. Horsnell, P. Rice, M.-A. Rajan- 
dream, and B. Barrell. Artemis: sequence visualization and annotation. Bioinformatics, 16(10):944-945, 2000.

[33] A. Smit, R. Hubley, and P. Green. Repeatmasker open-3.0. http://www.repeatmasker.org, 1996-2010.

[34] L. Stein et al. Genome annotation: from sequence to biology. Nature reviews genetics, 2(7):493-503, 2001. 


\section{Appendix A}

\section{Example GenBank data file}

Below is an example of a sequence entry data file in GenBank [30]. The example only contains two entries. In the real files there exists far more.

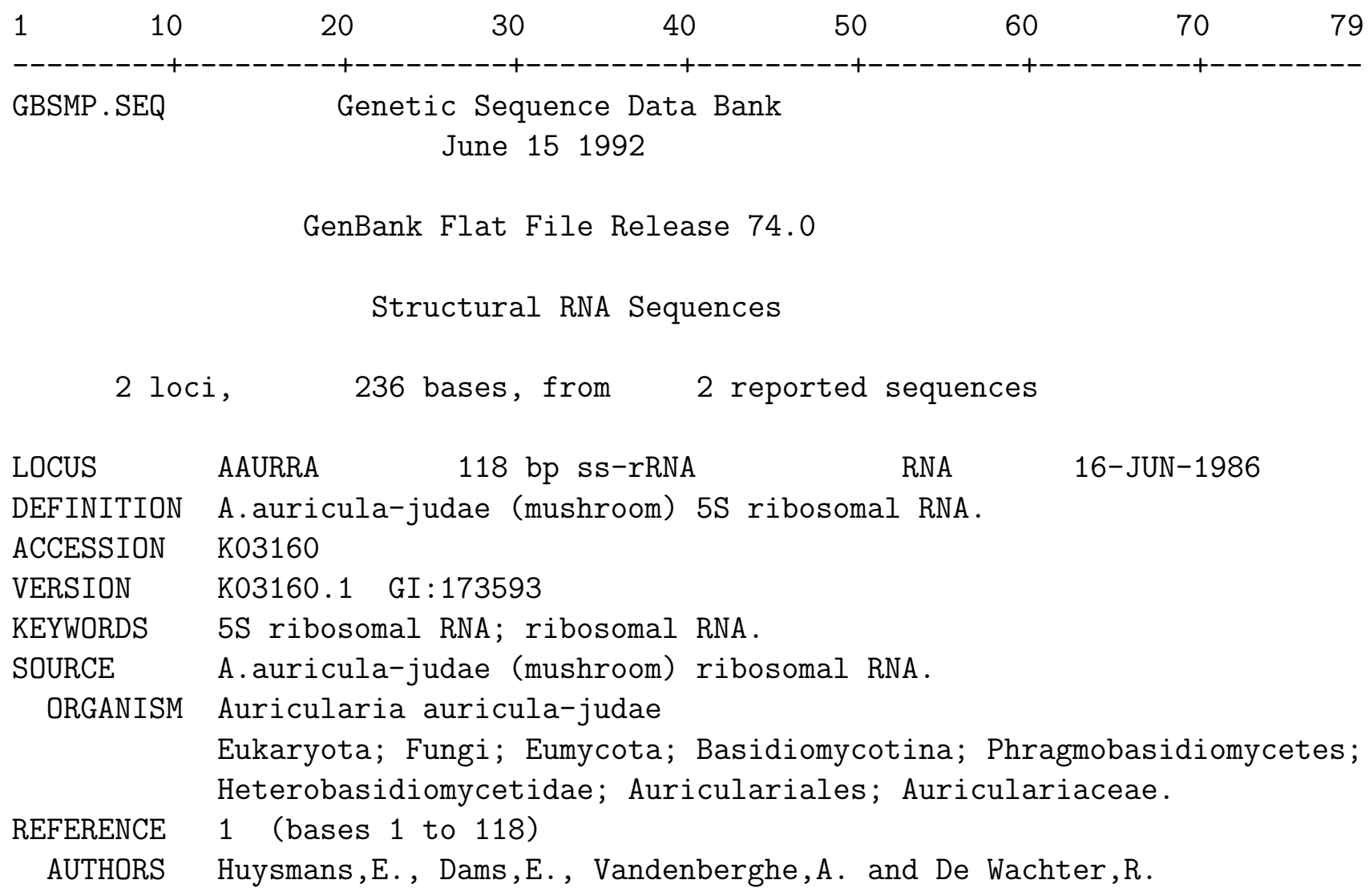


TITLE The nucleotide sequences of the 5S rRNAs of four mushrooms and their use in studying the phylogenetic position of basidiomycetes among the eukaryotes

JOURNAL Nucleic Acids Res. 11, 2871-2880 (1983)

FEATURES

Location/Qualifiers

rRNA

$$
\begin{aligned}
& 1 . .118 \\
& \text { /note="5S ribosomal RNA" }
\end{aligned}
$$

BASE COUNT $\quad 27 \mathrm{a} \quad 34 \mathrm{c} \quad 34 \mathrm{~g} \quad 23 \mathrm{t}$

ORIGIN 5' end of mature rRNA.

1 atccacggcc ataggactct gaaagcactg catcccgtcc gatctgcaaa gttaaccaga

61 gtaccgccca gttagtacca cggtggggga ccacgcggga atcctgggtg ctgtggtt

\section{//}

LOCUS

DEFINITION

ACCESSION

VERSION

KEYWORDS

SOURCE

ORGANISM

ABCRRAA

$118 \mathrm{bp}$ ss-rRNA

RNA

15-SEP-1990

Acetobacter sp. (strain MB 58) 5S ribosomal RNA, complete sequence. M34766

M34766.1 GI:173603

5S ribosomal RNA.

Acetobacter sp. (strain MB 58) rRNA.

Acetobacter sp.

Prokaryotae; Gracilicutes; Scotobacteria; Aerobic rods and cocci; Azotobacteraceae.

REFERENCE 1 (bases 1 to 118)

AUTHORS Bulygina,E.S., Galchenko,V.F., Govorukhina,N.I., Netrusov,A.I., Nikitin,D.I., Trotsenko,Y.A. and Chumakov,K.M.

TITLE Taxonomic studies of methylotrophic bacteria by $5 \mathrm{~S}$ ribosomal RNA sequencing

JOURNAL J. Gen. Microbiol. 136, 441-446 (1990)

FEATURES

rRNA

Location/Qualifiers

1 . 118

/note="5S ribosomal RNA"

BASE COUNT

27 a $40 \mathrm{c}$

$32 \mathrm{~g}$

$17 t$

2 others

ORIGIN

1 gatctggtgg ccatggcggg agcaaatcag ccgatcccat cccgaactcg gccgtcaat

61 gccccagcgc ccatgatact ctgcctcaag gcacggaaaa gtcggtcgcc gccagayy

//

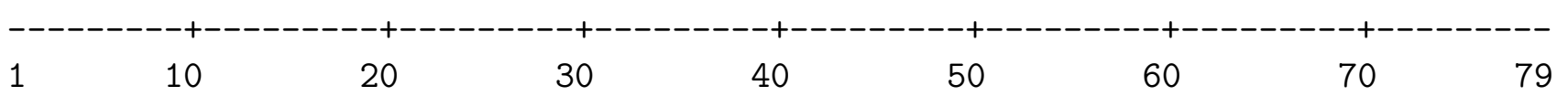

Example 1. Sample Sequence Data File 


\section{Appendix B}

\section{More information on the Chado}

\section{schema}

\section{B.1 Chado Structure}

The Chado schema is sub-divided into nine modules. Each module represents a specific idea. For example, the pub module which defines everything about a publication. The modules and their dependicies are represented in Figure B.1.

\section{B.2 Example graph using the Chado genetics module}

The following figure depicts how ontologies are used in the Chado schema. This example uses the genetics module. In this example, the data being depicted is the Allele phenotype alpha-spec ${ }^{\mathrm{rg} 41}$. The ontology starts with the gcontext record which defines the gene. The gcontext record links to a phenstatement 


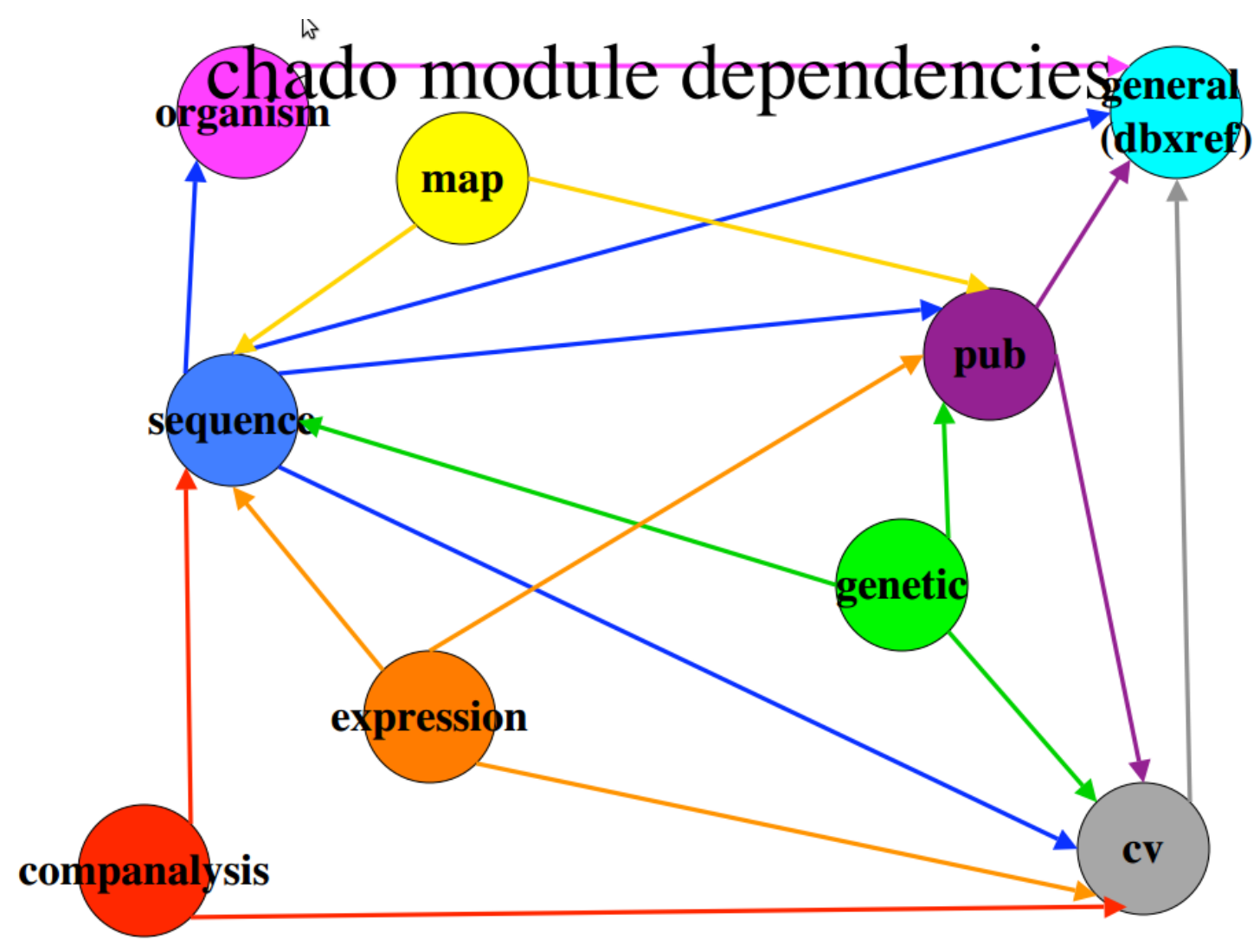

Figure B.1: Graph of the dependencies between the Chado modules [23].

record which defines the phenotype class of the allele alpha-spec ${ }^{\text {rg } 41}$. Specifically that the gene is lethal, larval, and recessive. All of the previous terminology link to separate cvterm records which define the common terminology used through the Chado schema. 
Allele phenotype alpha-spec ${ }^{\text {rg } 41}$

Phenotypic class:

lethal I larval I recessive
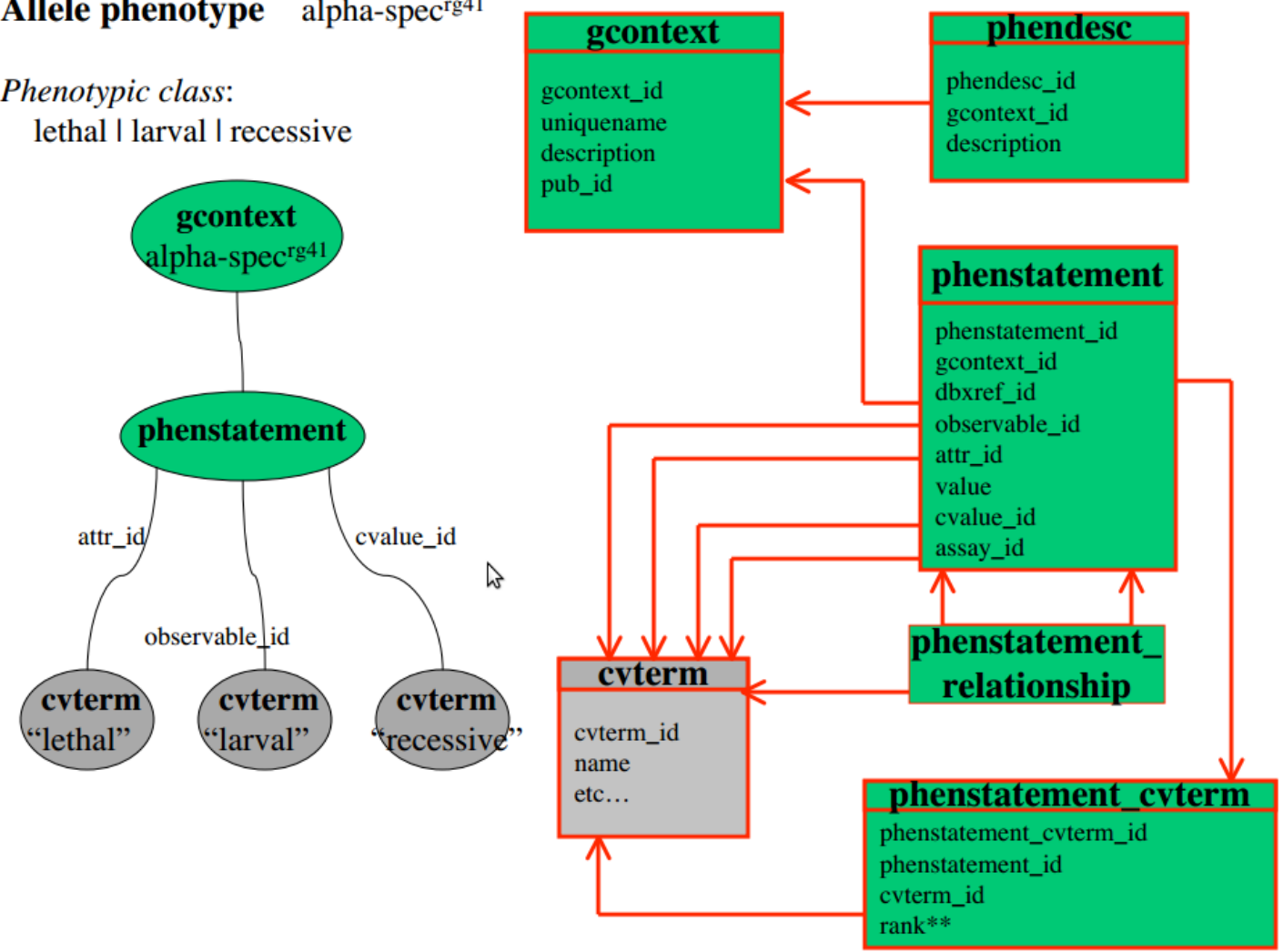

Figure B.2: Example Graph of Data in the Chado Genetics Module [23]. 


\section{Appendix C}

\section{SQL create table statements for}

\section{$C G A T$}

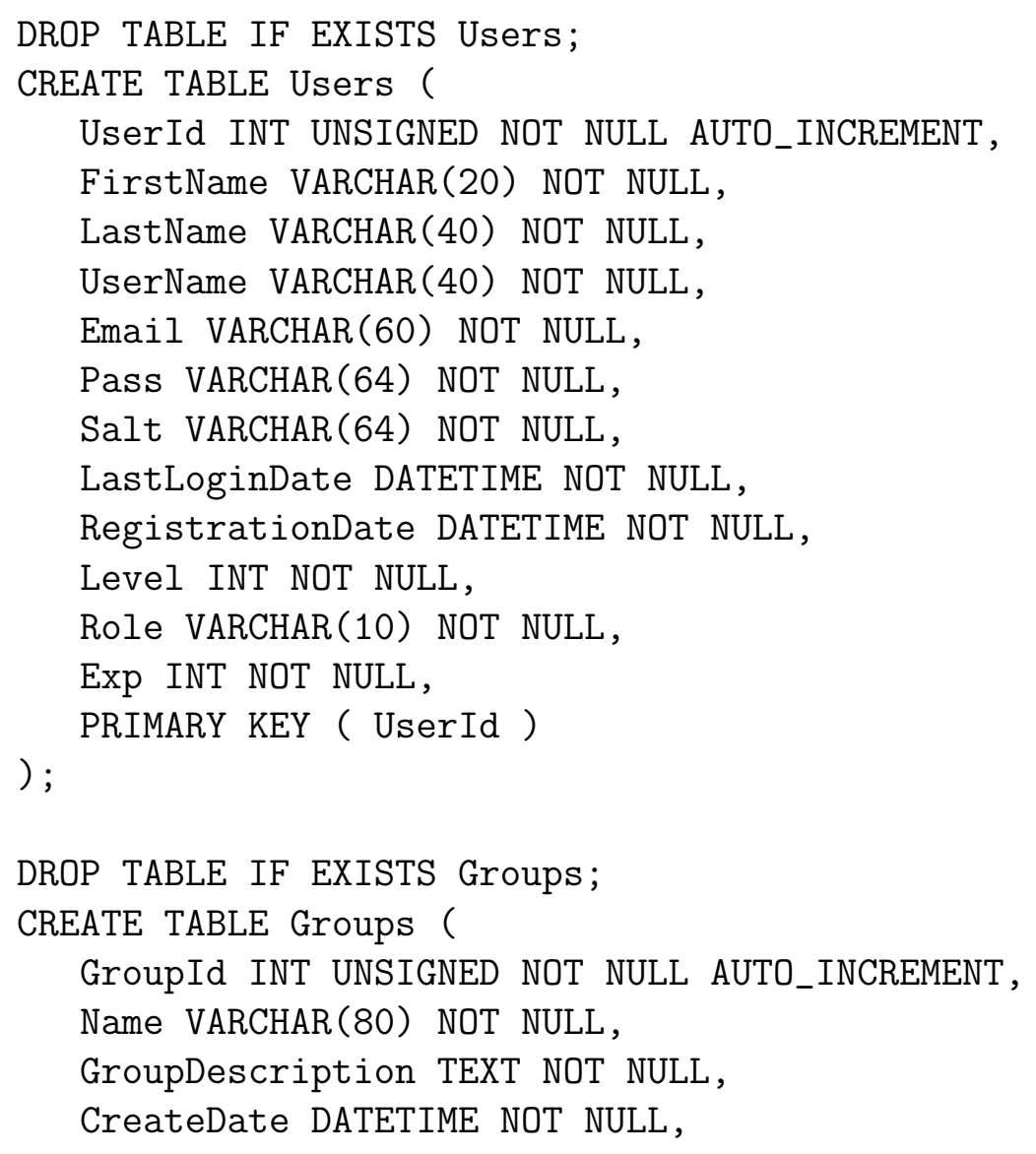




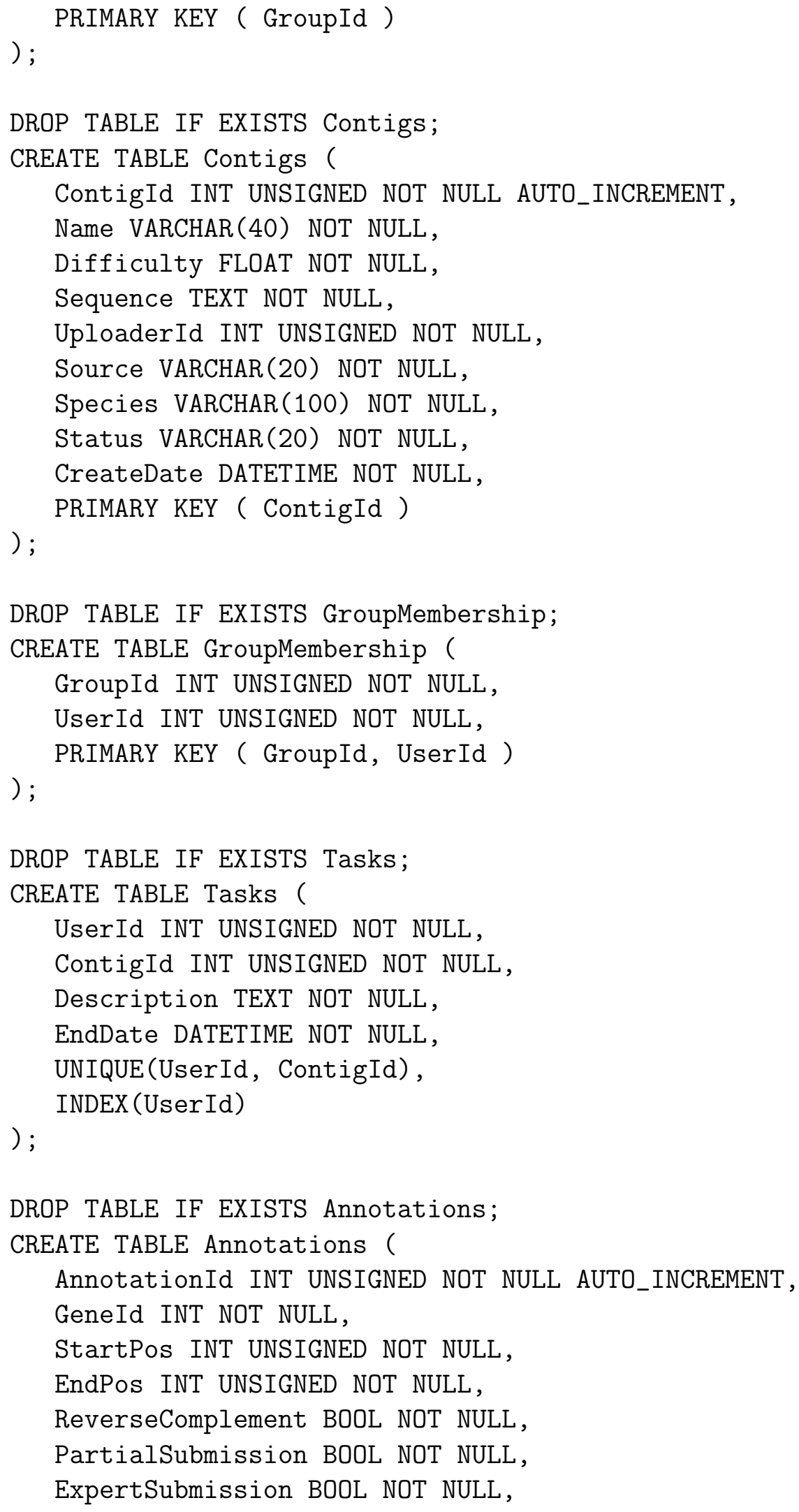




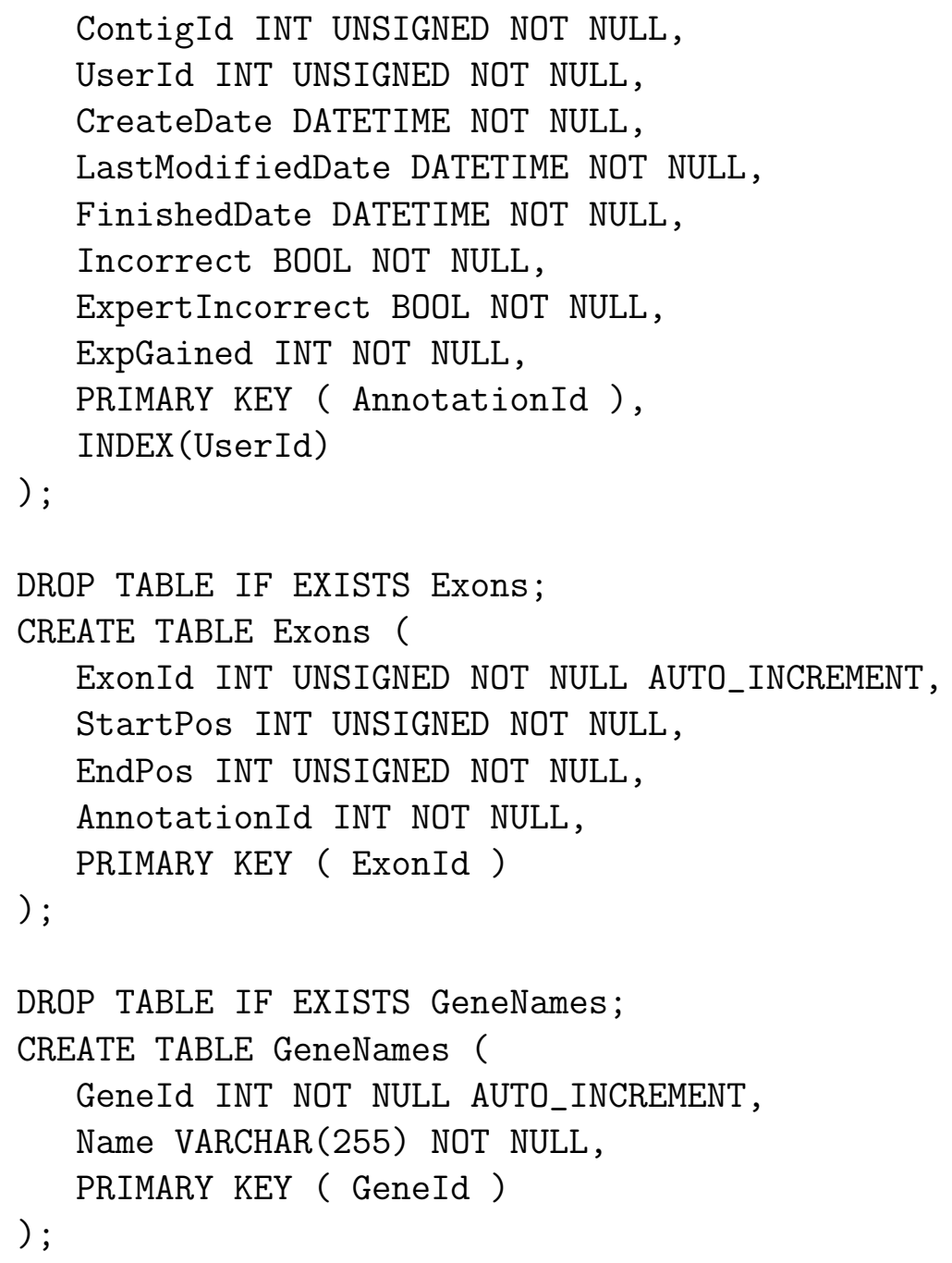




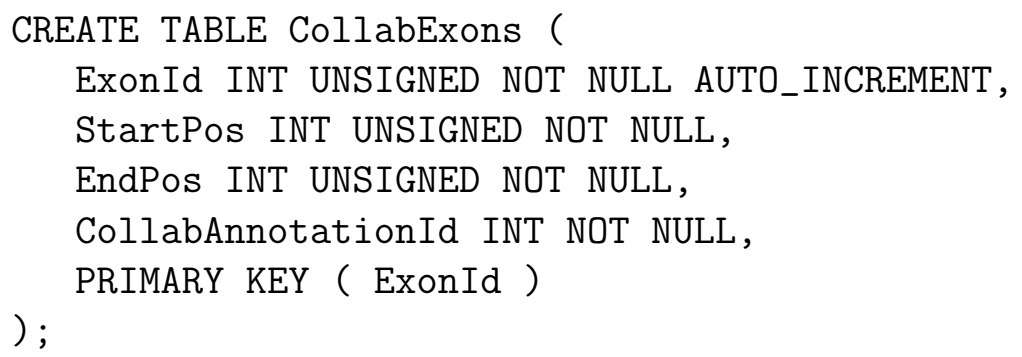

DROP TABLE IF EXISTS ReadWriteTest;

CREATE TABLE ReadWriteTest(id INT, name VARCHAR(128)); 